\title{
Prediction of the plastic anisotropy of magnesium alloys with synthetic textures and implications for the effect of texture on formability
}

\author{
Victoria M. Miller, ${ }^{\mathrm{a}, *}$, Tracy D. Berman ${ }^{\mathrm{b}}$, Irene J. Beyerlein ${ }^{\mathrm{c}}$, J. Wayne Jones ${ }^{\mathrm{b}}$, Tresa M. Pollock ${ }^{\mathrm{a}}$ \\ ${ }^{a}$ Materials Department, University of California Santa Barbara, Santa Barbara, CA 93106-5050 \\ ${ }^{b}$ Materials Science and Engineering Department, 2300 Hayward Street, Ann Arbor, MI 48109 \\ ${ }^{c}$ Theoretical Division, Los Alamos National Laboratory, Los Alamos, NM 87545
}

\begin{abstract}
Crystallographic texture is a well-known microstructural feature influencing the formability of magnesium alloys. However, the effects of individual texture characteristics common after thermomechanical processing have not been isolated due to the experimental challenge associated with varying them independently. Similarly, the effect of the propensity for twinning on formability, which both accommodates deformation and reorients the crystal, have not been systematically studied. This study uses synthetic sheet textures in conjunction with a viscoplastic self-consistent (VPSC) polycrystal plasticity model to predict deformation and formability behavior. The VPSC model was first parameterized based on experimental mechanical data and textures from fine-grained thixomolded and thermomechanically processed AZ61L. Subsequently, synthetic textures were generated to examine the effects of basal peak intensity, prismatic plane distribution, and asymmetry of the basal pole figure peak. Of these texture characteristics, basal peak strength is the most important predictor of forming behavior, with prismatic plane distribution and c-axis anisotropy resulting in comparatively weak effects. In the second part of the study, the effective critical resolved shear stress for twinning was varied, resulting in poorer forming behavior with easier twin activation. In both cases, increasing prismatic slip activity was deleterious to the predicted forming behavior.
\end{abstract}

Keywords: magnesium alloys, viscoplastic self-consistent model, formability, texture

\section{Introduction}

The commercial adoption of wrought magnesium alloys has been limited, largely because conventional magnesium alloys have limited formability at room temperature. Formability in these materials is dictated

\footnotetext{
*Corresponding author

Email address: vmiller@umail.ucsb.edu (Victoria M. Miller)
} 
by a complex array of factors, including grain size, second phase particle distribution, and alloy composition. However, the dominant factor in many cases is crystallographic texture [1]. Magnesium alloys typically develop strong basal deformation textures, with the c-axes of individual grains aligning approximately parallel to the sheet normal direction in rolled material [2]. This texture is the result of the relative ease of slip on the basal system in comparison to the prismatic and pyramidal slip systems, and its development is further aided by the activity of extension twinning. The basal texture in conventional alloys typically persists after recovery and recrystallization heat treatment and strengthens during subsequent grain growth [3-5].

A strong basal texture is highly detrimental to formability because the grain orientations are unfavorable for the easiest deformation mode, basal slip. Gehrmann et al. have reported that materials with a strong basal texture are more susceptible to strain localization and the formation of shear bands [6]. Another study on strongly textured polycrystals mechanically tested in various orientations revealed the striking plastic anisotropy that results from strong basal textures [7]. Numerous studies have also noted that increasing peak intensity of the basal pole figure is correlated with reduced formability [8-11]. The planar anisotropy of the basal pole (asymmetry of the basal peak) figure common in textured magnesium materials has also been noted, though its effects on forming behavior have not been fully explored [12, 13].

In addition, forming behavior is sensitive to relative deformation mode activity; it will be especially sensitive to twin mode activity because twinning not only accommodates deformation, but also contributes to plastic anisotropy and accelerates texture evolution by reorienting the crystal. Modification of the relative activity of the extension twin commonly observed in magnesium alloys has been reported both due to changes in alloying and grain size. In particular, rare earth alloy additions have been reported to greatly strengthen the extension twinning mode as well as activating other twin modes [14-18]. Additionally, decreasing grain size has been reported to very strongly inhibit tensile twinning [19-21] or to coincide with a transition to slip dominated flow [22-24]. However, the role of twin mode strength in determining formability remains unclear due to the complex interdependence of alloying, microstructural features, and deformation mode activity.

Experimental investigation of formability in magnesium alloys has generally relied on the measurement of r-values, also known as Lankford coefficients. The r-values are defined as the ratio between the strains in the width and thickness directions after a tensile test to a pre-defined strain threshold [25]. This ratio is calculated for three or more specimen orientations in the sheet plane, usually with the tensile axis angled by $0^{\circ}, 45^{\circ}$, and $90^{\circ}$ from the rolling direction. An average r-value, $\bar{r}$, can be calculated as:

$$
\bar{r}=\frac{\left(r_{0}+2 r_{45}+r_{90}\right)}{4}
$$




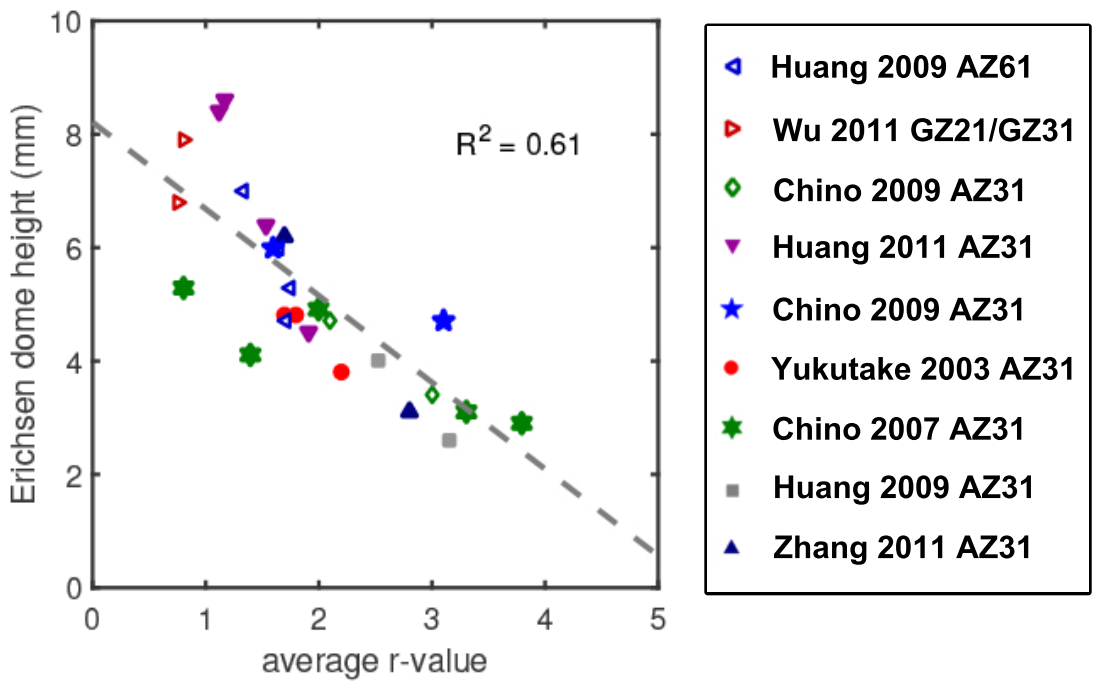

Figure 1: Correlation between Erichsen biaxial stretch test maximum dome height and the value of $\bar{r}$. Data obtained from the literature [27. 35]. Plot adapted from Berman et al. [10].

A survey of the available literature for magnesium alloys, adapted from Ref. [10] and shown in Figure 1$]$ shows that $\bar{r}$ has a negative linear correlation with the maximum dome height achieved in a standard Erichsen biaxial stretch test, indicating that $\bar{r}$ is a valid indicator of room temperature formability. Values of $\bar{r}$ greater than 3.0 are common in commercial Mg alloys [7, 26], but the best forming behavior is observed in alloys that have $\bar{r}$ values near 1.0.

While the detrimental effect of strong basal textures on r-values is well-established, the influence of other texture characteristics commonly produced by magnesium processing paths has not been systematically examined. This is due, at least in part, to the inability to experimentally vary texture independent of other parameters important to formability [11, 36]. In addition to texture, r-values have been demonstrated to be sensitive to grain shape [37, 38], grain size [36, 39], second phase particles [40], and dislocation content [41]. Each of these microstructural features can have interactive effects with crystallographic texture during thermomechanical processing. Polycrystal plasticity simulations offer the opportunity to isolate particular texture characteristics or deformation mode activities while guaranteeing that all other material and deformation parameters are held constant.

A number of researchers have utilized polycrystal plasticity tools to examine formability. Forming limit diagrams have been computed using several different polycrystal frameworks in cubic metals (e.g. [38, 42, 43]) and in hep alloys, including Mg [44-46]. In particular, Wang et al. [44] found significantly improved 
formability with decreasing basal peak strength by simulating forming tests on the same texture in different orientations. Additionally, Neil et al. [46] compared the formability of magnesium having a random texture with material having an experimentally measured rolling texture, again highlighting the importance of weak intensity of the basal peak for enhancement of formability. Others have used polycrystal plasticity models to calculate r-values, either using the ratio of instantaneous width and thickness strain rates at yield [47] or the more conventional ratio of width and thickness strains after a fixed amount of deformation [13, 26]. In particular, Agnew et al. [13, 26] have examined possible sources of plastic anisotropy of magnesium alloy sheet, concluding that the anisotropy is largely attributable to the texture.

In this work, the viscoplastic self-consistent (VPSC) framework developed by Lebensohn and Tomé [47, 48] is used to simulate uniaxial tension tests at room temperature, and the effect of various textures and twin mode strengths are considered. To examine the effect of varied texture characteristics, the single crystal hardening parameters are held constant, with deformation behavior parameterized to emulate a fine-grained thixomolded and thermomechanically processed (TTMP) AZ61L magnesium alloy, as described in Ref. [10]. The effect twin mode strength on deformation behavior is then considered for different starting textures. For all examined textures and twin mode strengths, increased activation of prismatic slip corresponded to higher r-values, suggesting poorer forming behavior.

In the first section of this paper, VPSC r-values calculated via several different methods are compared to experimental values from TTMP AZ61L for validation of the technique. The second section examines synthetic textures to isolate texture characteristics common in rolled Mg alloys, namely basal peak intensity, symmetry of the prismatic plane distribution, and basal peak symmetry. In the third section of the paper, the changes in deformation behavior with varied strength of the extension twinning mode are examined. The changes in stress-strain behavior, slip system activity, and r-value indicators of formability are presented. These results are then discussed in the context of the relative importance of each deformation mode for formability, particularly prismatic slip.

\section{Experimental and Simulation Procedures}

\subsection{Experimental Material Used for Parameterization}

The material used in this study to parameterize the VPSC model is thixomolded and thermomechanically processed (TTMP) AZ61L, which was part of a larger study on microstructural evolution during processing [10]. The key aspects relevant to this study are described here. 


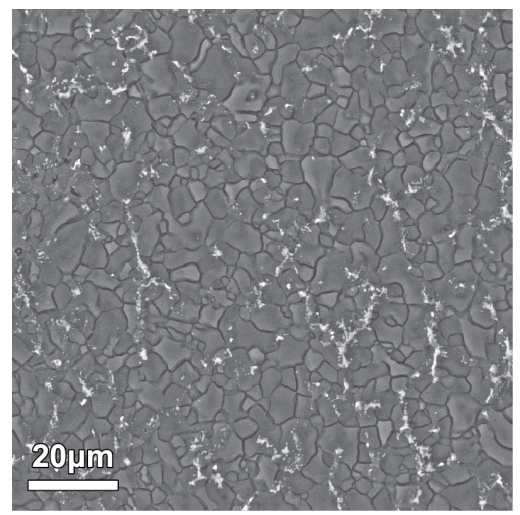

Figure 2: Secondary electron image of the microstructure of the AZ61L material used for parameterization of the VPSC model. The rolling direction is vertical and the sheet normal is out of the plane of the page.

The AZ61L alloy was thixomolded into 3mm thick plates by nanoMAG, LLC utilizing molding parameters that result in a solid fraction of less than $5 \%$. These plates were then preheated for 5 minutes at $588 \mathrm{~K}$ $\left(315^{\circ} \mathrm{C}\right)$ and warm rolled (roll temperature approximately $\left.473 \mathrm{~K}\left(200^{\circ} \mathrm{C}\right)\right)$ to a $40 \%$ reduction in thickness in a single pass. The rolled sheets were allowed to air-cool to the ambient temperature. Dogbone tensile specimens (subsize specimen designation in ASTM E8/E8M-11) were machined from the sheet with the tensile axes along both the rolling direction, transverse direction, and inclined by $45^{\circ}$ to the $\mathrm{RD}$ in the sheet plane. The machined specimens were heat treated for 10 minutes at $558 \mathrm{~K}\left(285^{\circ} \mathrm{C}\right)$, resulting in a fine-grained recrystallized structure [49] as shown in Figure 2] After heat treatment, the $\alpha$-Mg grain size is $3.1 \mu \mathrm{m}$, and the volume fraction of the $\beta-\mathrm{Mg}_{17} \mathrm{Al}_{12}$ is $5 \%$ [10].

The heat-treated dogbone specimens were tensile tested at room temperature using an Instron 5505B load frame. The tests were conducted at a constant crosshead speed of $0.01 \mathrm{~mm} / \mathrm{s}$, which is equivalent to an initial strain rate of $4 \times 10^{-4} \mathrm{~s}^{-1}$, to a total plastic strain of $10 \%$ to calculate the r-values.

Metallographic specimens for secondary electron microscopy and electron backscatter diffraction (EBSD) were prepared from the sheet midplane using standard techniques, with $1 \mu \mathrm{m}$ diamond paste as the final polishing step. During all polishing steps, $\mathrm{MetaDi}^{\circledR}$ fluid was used as a lubricant. Specimens for secondary electron imaging were etched for $3 \mathrm{~s}$ with a solution containing $10 \mathrm{~mL}$ water, $10 \mathrm{~mL}$ acetic acid, $4.3 \mathrm{~g}$ picric acid, and $70 \mathrm{~mL}$ ethanol. Samples used for EBSD were instead etched with a solution containing $60 \mathrm{~mL}$ ethanol, $20 \mathrm{~mL}$ water, $15 \mathrm{~mL}$ glacial acetic acid, and $5 \mathrm{~mL}$ of nitric acid held at $5^{\circ} \mathrm{C}$. Texture measurements were performed via EBSD on scan areas sampling approximately 10,000 grains. 


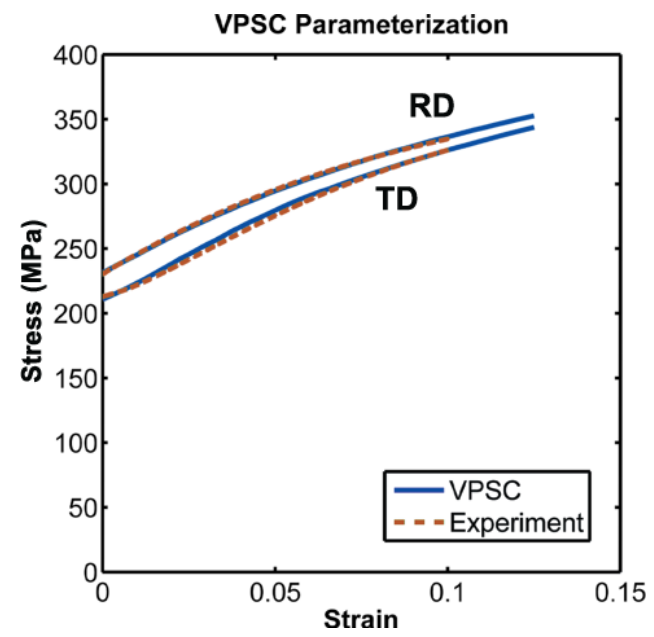

Figure 3: A comparison of the experimental and VPSC-simulated tensile tests along both the RD and TD. Adapted from Ref. [50].

\subsection{VPSC Simulation and Parameterization}

Simulations of the plastic response of these initially textured materials were conducted using the viscoplastic self-consistent approach [47]. Within this framework each grain is considered as an Eshelby-type inclusion in a homogeneous effective medium with properties of the aggregate polycrystal. A macroscopic deformation is imposed and shear rates are calculated on the relevant slip or twinning systems for each grain. These shear rates are used to calculate crystallographic texture evolution, grain-scale hardening, slip activity, macroscopic stress-strain behavior, and r-values. All simulated mechanical tests in this work are uniaxial tension tests along the stated material direction at a strain rate of $10^{-3} \mathrm{~s}^{-1}$.

An extended Voce law is used to describe the hardening behavior of each slip or twinning system, where the critical resolved shear on system $s$ after strain $\Gamma$ is given by:

$$
\tau^{s}=\tau_{0}^{s}+\left(\tau_{1}^{s}+\theta_{1}^{s} \Gamma\right)\left(1-\exp \left(-\frac{\theta_{0}^{s} \Gamma}{\tau_{1}^{s}}\right)\right)
$$

[51] in which the $\tau_{i}^{s}$ and $\theta_{i}^{s}$ values are phenomenological hardening parameters for the $s^{\text {th }}$ slip or twinning system. The $\tau_{0}$ parameter can be considered an effective initial critical resolved shear stress. Twinning is captured using the Predominant Twin Reorientation scheme proposed by Tomé et al. [52]. This twinning scheme is a Schmid-type law that accounts for the directionality of twinning and also accounts for the effect of twin activity on texture. Reorientation occurs when twinning contributes $10 \%$ of the strain in a given grain. Hardening parameters for each deformation mode were selected to reproduce the hardening behavior of thixomolded AZ61L after rolling and complete recrystallization at $285^{\circ} \mathrm{C}$, as presented in Reference [10]. A 
Table 1: Voce hardening parameters utilized in all subsequent VPSC simulations.

\begin{tabular}{lcccccc}
\hline & & $\tau_{0}(\mathrm{MPa})$ & $\tau_{1}(\mathrm{MPa})$ & $\theta_{0}$ & $\theta_{1}$ & Note \\
\cline { 3 - 6 } basal & $\{0001\}\langle 2 \overline{11} 0\rangle$ & 63 & 20 & 190 & 100 & \\
prismatic & $\{10 \overline{1} 0\}\langle 2 \overline{11} 0\rangle$ & 165 & 20 & 300 & 90 & \\
pyramidal & $\{1 \overline{1} 2\}\langle\overline{11} 23\rangle$ & 230 & 20 & 230 & 90 & \\
extension twin & $\{10 \overline{1} 2\}\langle\overline{1} 011\rangle$ & 200 & 0 & 100 & 30 & \\
& & 110 & 0 & 100 & 30 & matched with TTMP AZ61L \\
& & 70 & 0 & 100 & 30 & \\
\hline
\end{tabular}

comparison of simulated and experimental tensile tests along the sheet rolling direction (RD) and transverse direction (TD) are shown in Figure 3. The Voce hardening parameters are presented in Table 1. Basal, prismatic, pyramidal, and extension twinning deformation modes are allowed. These hardening parameters were originally used in the VPSC simulations in Ref. [50]. The experimentally observed plastic anisotropy is reproduced in the RD vs TD, as evidenced by the good matching between the VPSC and experimental stress-strain curves. In addition, two other $\tau_{0}$ values were examined for the extension twin mode, as shown in Table 1. The values were selected based on the basal:twin CRSS ratios reported in the literature, which is discussed further in Section 4.1

The experimental and simulated texture evolution after $10 \%$ tensile strain tested along the (RD) and (TD) is presented in Figure 4. During uniaxial tension along the RD, in both the simulation and experiment the basal poles spread toward the sheet TD, while the prismatic poles align along the RD. Conversely, during tension along the sheet TD, the basal poles align with the RD while the prismatic poles preferentially align with the loading direction. In both cases, the VPSC model over-predicts texture evolution; this effect is common and has been discussed elsewhere in the literature [53 57]. Overall, the good qualitative match in texture evolution suggests that the model is adequately capturing the balance of active deformation mechanisms. This is further verified by the excellent match between the experimentally determined and VPSC simulated r-values presented in Figure 5. The simulated r-values are calculated as the ratio of accumulated width and thickness strains after a simulated tensile test to $10 \%$ elongation (i.e. in a manner identical to the experimental procedure). These hardening parameters are used for all other VPSC simulations presented in Section 3.1. The experimentally-verified hardening parameters for slip are also used in Section 3.2, while the twin mode strength is varied. 
Starting Texture

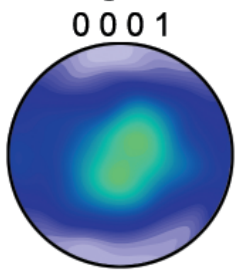

$10 \overline{10}$

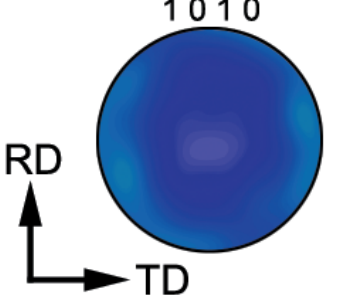

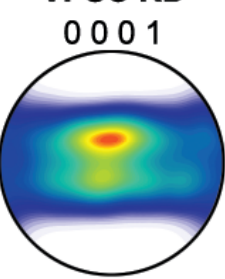

Exp. RD

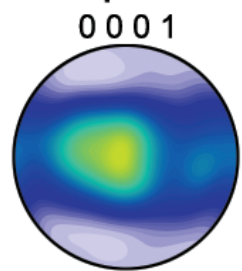

$10 \overline{10}$
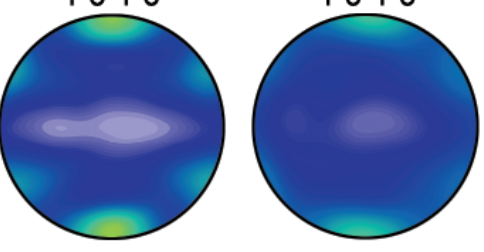

VPSC TD 0001

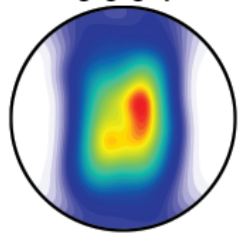

$10 \overline{1} 0$

Exp. TD

0001

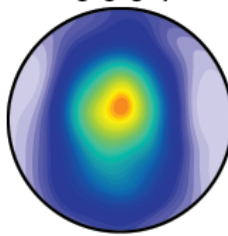

$10 \overline{10}$

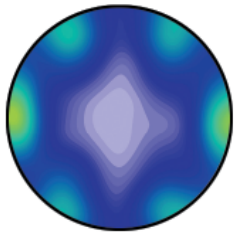

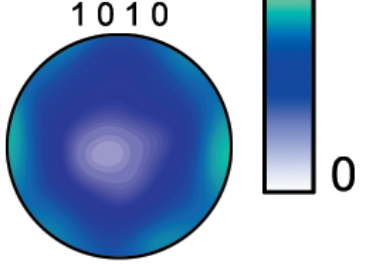

Figure 4: Comparison of experimental and VPSC-predicted textures after uniaxial tensile deformation along the transverse direction (TD) and rolling direction (RD).

Table 2: Synthetic microstructure generation DREAM.3D pipeline

\begin{tabular}{lll}
\hline Filter \# & Filter Name & Filter Purpose \\
\hline 1 & Read DREAM.3D Data File & Load goal statistics created by Stats Generator \\
2 & Initialize Synthetic Volume & Create empty volume \\
3 & Establish Shape Types & Assigns shape type (ellipsoid) to grains \\
4 & Pack Primary Phases & Generates individual grains inside empty volume \\
5 & Find Feature Neighbors & Determines number and identity of neighbors for each grain \\
6 & Match Crystallography & Assigns orientations to match orientation density function (ODF) \\
7 & Generate IPF Colors & Generates inverse pole figure colors for each grain \\
8 & Write DREAM.3D Data File & Writes all microstructure attributes to DREAM.3D data file \\
9 & Write Los Alamos FFT File & Writes text file containing voxel locations and orientations \\
\hline
\end{tabular}

\subsection{Synthetic Texture Generation}

Synthetic textures were generated for analysis using DREAM.3D Version 5.2 and the Stats Generator auxiliary tool [58]. First, Stats Generator was used to define the target texture and create a starting DREAM.3D data file. A DREAM.3D pipeline that uses the target statistics output by Stats Generator is presented in Table 2. Note that this pipeline generates complete three-dimensional microstructures, including information such as grain neighbors, shapes, and size distribution, appropriate for a more rigorous (but computationally costly) crystal plasticity finite element modeling approach. Only the weighted grain orientations were used for the VPSC simulation in this work.

The synthetic volume created was a cube 150 voxels on edge, with a voxel spacing of $0.5 \mu \mathrm{m}$ in all di- 


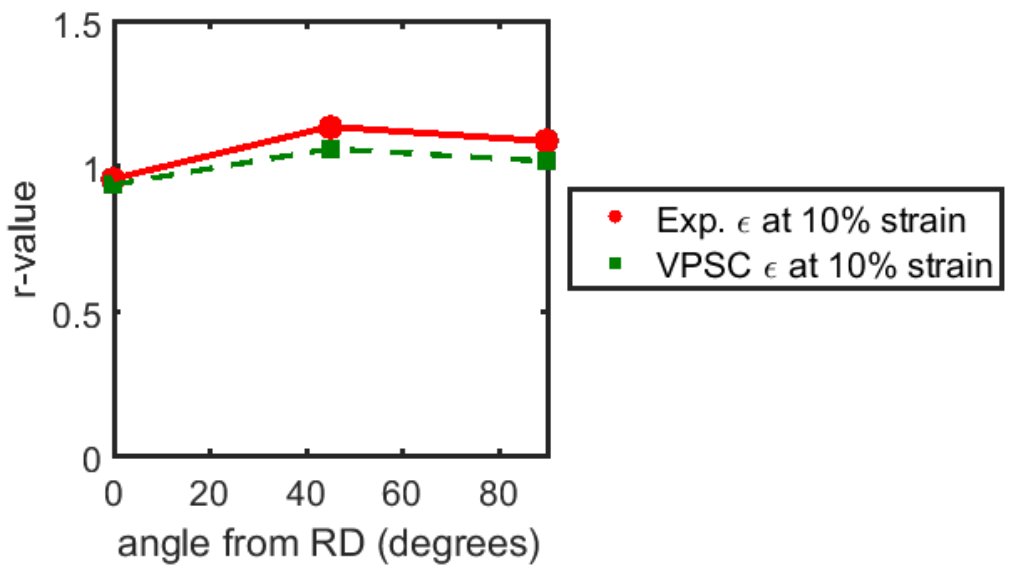

Figure 5: Comparison of simulated and experimental r-values at $10 \%$ strain.

rections. This resulted in volumes containing more than 30,000 grains. The MTEX texture analysis toolbox for Matlab [59] was used to import the DREAM.3D-output text files as orientation density functions (ODFs) and output VPSC texture files with 10,000 grains.

In Section 3.1, various texture characteristics, namely basal peak intensity, symmetry of the prismatic plane distribution, and basal peak symmetry are isolated and systematically varied to examine their effects on formability. For each texture characteristic, two to three textures are generated and VPSC was used to simulate tensile tests at a constant strain rate of $10^{-3} / \mathrm{s}$ using the single crystal hardening parameters described in Section 2.2. In Section 3.2, the effect of varied strength of the extension twinning mode on r-value behavior is examined while leaving the CRSS values for slip constant. In both sections, the changes in stress-strain response, relative slip activity, r-values, and $\bar{r}$ evolution with strain are reported.

\section{Results}

\subsection{Varied Texture Characteristics}

\subsubsection{Basal Peak Strength}

First, the effect of varied basal peak intensity on formability was isolated for the fine grain size described in Table 1. Three relatively strong basal textures were compared, as shown in Figure 6 The differences in deformation behavior are shown in Figure 7 As shown in the stress-strain curves in Figure 7 , deformation along the RD and TD is very similar due to the single symmetrical peak in the basal pole figure. Slight differences are observed, particularly above approximately $8 \%$ tensile strain, due to the sixfold symmetry of the prismatic pole figure. 


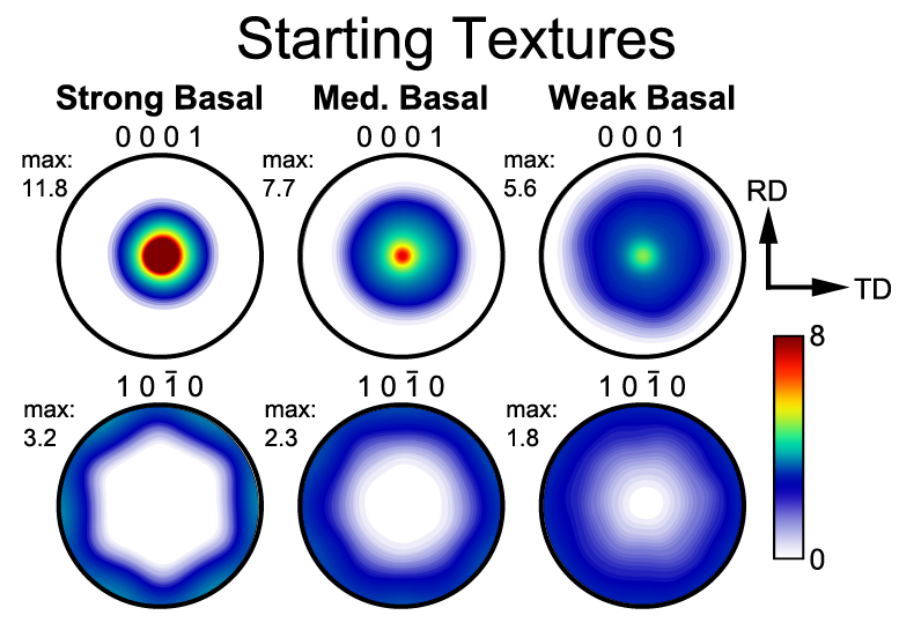

Final Textures

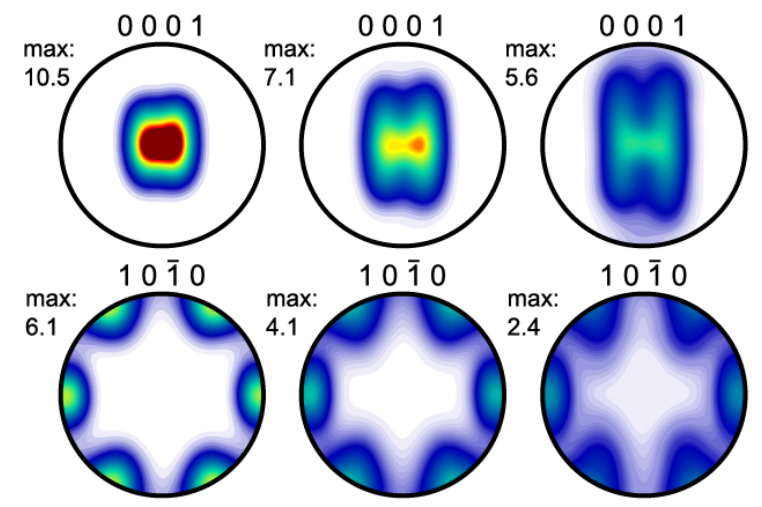

PF Difference Maps

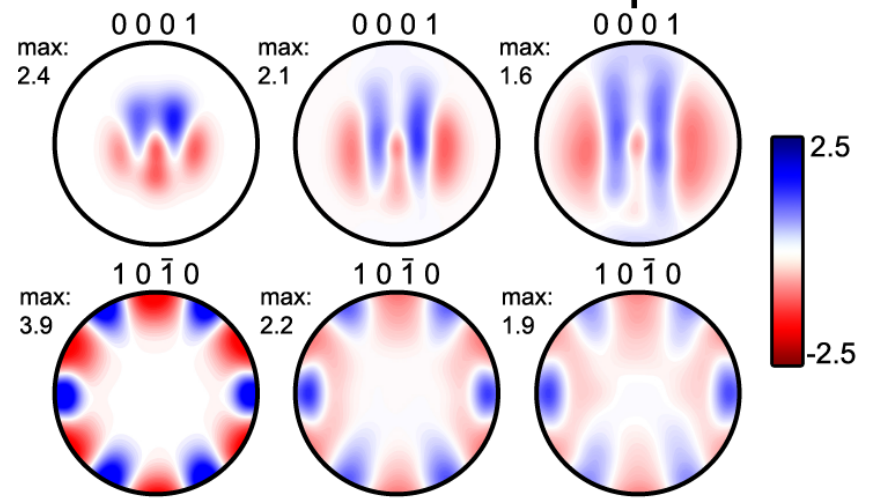

Figure 6: Initial textures, final textures, and pole figure difference maps during uniaxial tension along the sheet transverse direction to $20 \%$ strain. 
RD Uniaxial Tension

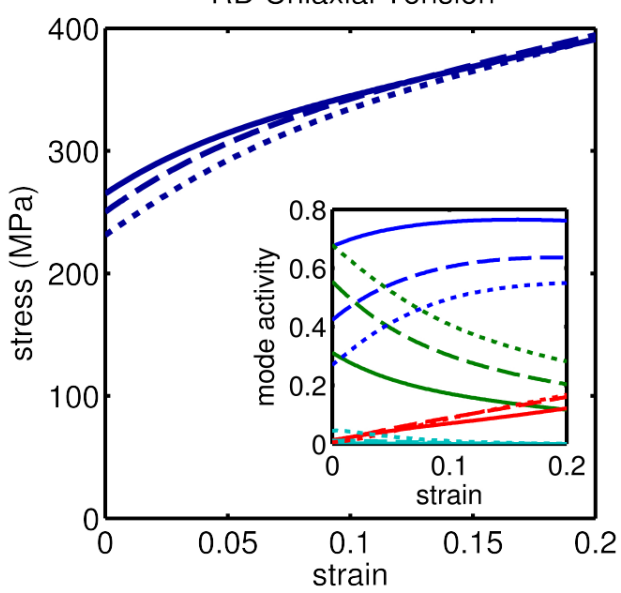

Lankford Values at 10\% Strain

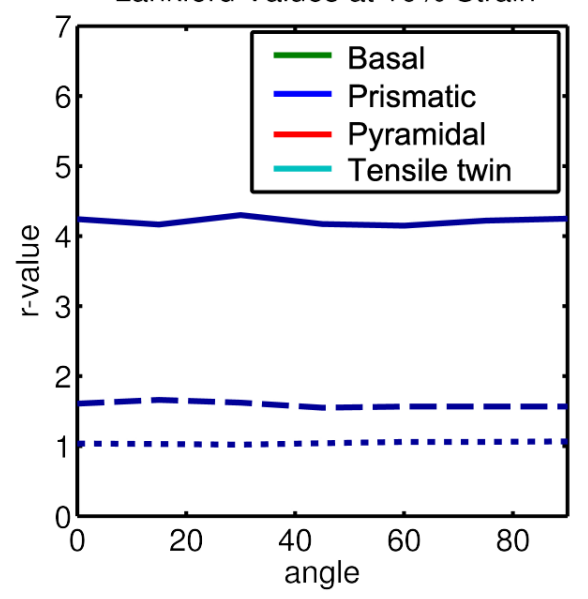

TD Uniaxial Tension

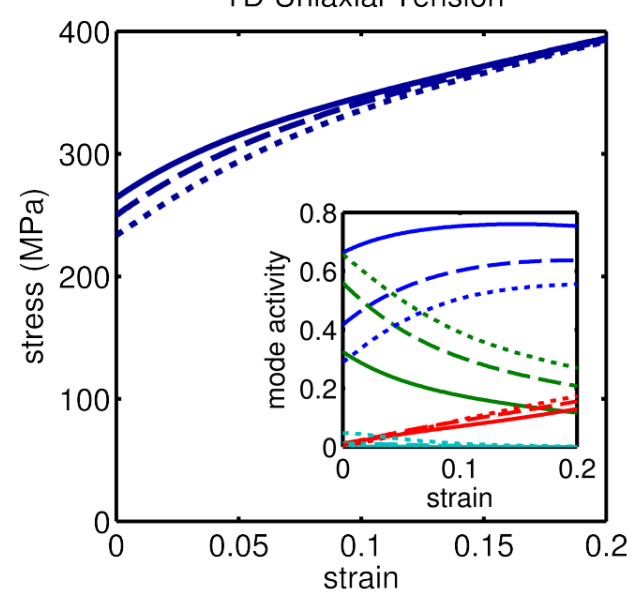

$\bar{r}$

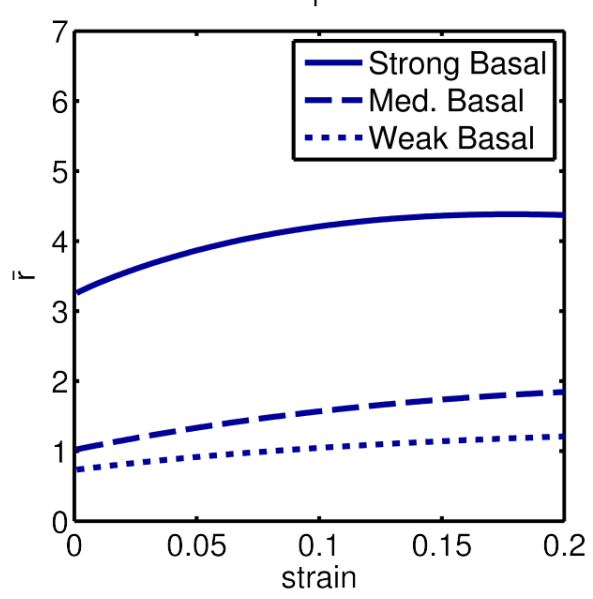

Figure 7: Comparison of deformation behavior of fine-grained sheet with varied basal peak intensity. 
The texture evolution during uniaxial tension to $20 \%$ elongation is presented in Figure 6 . The initial textures, deformed textures, and pole figure difference plots are shown. For all three basal textures, the peak intensity of the basal pole figure slightly decreased during deformation, while the maximum value of the prismatic pole figure increased. The pole figure difference plots show that the basal poles spread toward the TD during deformation and the basal peak starts to split toward the RD. The spreading of the basal peak is more pronounced for weaker basal textures. The strengthening of the sixfold symmetry of the prism pole figure is most pronounced for the strongest basal texture.

This texture evolution is reflected by the deformation mode activity, shown in insets in the stress-strain curves in Figure 7] Deformation during tension was dominated by a combination of basal and prismatic glide for all three basal textures, with some activation of pyramidal slip. The weakest basal texture also allowed for the activation of some tensile twinning during deformation, accounting for approximately $7 \%$ of deformation at yield. The primary effect of decreasing basal peak intensity is an increasing dominance of basal slip. Grains with perfectly basal orientations (c-axes parallel to the sheet normal direction) have a Schmid factor equal to zero for basal slip, so the harder prismatic slip mode must be active. This reliance on prismatic slip also accounts for the observed difference in macroscopic yield strength.

Strong variations in both the r-values and their evolution with strain were also predicted, as shown in Figure 77, The r-values increased with basal peak strength and remained approximately constant within the sheet plane. The stronger basal textures also underwent greater increases in $\bar{r}$ during straining.

\subsubsection{Varied Planar Anisotropy}

Figure 8 shows the effect of varied in-plane anisotropy on deformation behavior. The textures shown in Figure 9 vary in the amount of spread in the distribution of the c-axes toward the sheet transverse direction while maintaining a nearly constant basal peak intensity. Similar differences in texture are observed after deformation with varied fractions of intermetallic particles [60].

As shown in Figure 8, varied in-plane anisotropy results in minimal changes in tensile behavior along the rolling direction. Small differences in slip activity are present, with increasing planar anisotropy resulting in increased prismatic and pyramidal slip activity. Basal slip activity is slightly decreased, and no extension twinning is observed in any of the textures when loaded in tension along the rolling direction.

Preferred orientation of the in-plane c-axes results in anisotropy of the deformation behavior, particularly slip system activity. The change in yield strength is small, with increased anisotropy of the basal peak increasing the yield strength along the RD and decreasing it along the TD. This is a result of the dramatically increased activation of tension twins during TD strain with increasing anisotropy. When the texture has low 
TD spread and twinning is unavailable, additional prismatic slip accommodates strain in TD tension.

The varied planar anisotropy has only small effects on the measures of formability considered here in comparison to basal peak strength, with increased planar anisotropy resulting in slightly increased average r-values. For all three conditions, the r-values are between 1.0 and 2.0 for all in-plane orientations, resulting in $\bar{r}$ values near 1.5 .

\subsubsection{Varied Prismatic Symmetry}

The effect of sixfold symmetry in the prismatic pole figure on forming behavior is also considered. This sixfold symmetry occurs after deformation pathways where prismatic slip plays a strong role [10] and has also been reported after recrystallization and grain growth [3, 5]. However, the implications for forming behavior have not been discussed in detail. Two strong basal textures of approximately equal strength, shown in Figure 10, compare the cases of strong sixfold prism symmetry with an approximately isotropic in-plane distribution of a-axis directions (radial isotropy).

Minimal differences in deformation behavior are observed between the two textures. As shown in Figure 11 the stress-strain behavior of the two textures is nearly identical, with the predicted strengths varying by less than $3 \mathrm{MPa}$ after $20 \%$ tensile elongation in both the RD and TD configurations. This slight variation in flow stress is similar to that observed at high strains in the basal textures discussed in Section 3.1.1. The slip activity is nearly identical for both cases. The texture evolution, shown in Figure 10, is similar to the basal texture described in Section 3.1.1 with weakening of the basal peak and strengthening of the sixfold symmetry of the prismatic pole figure.

After $10 \%$ elongation, the r-values for both textures were high and approximately constant for all inplane orientations tested, falling between 4.0 and 4.5. These r-values are similar to the strong singly peaked basal texture discussed in the previous section where prismatic slip is dominant, as shown in the Figure 11 insets. Consequently, the $\bar{r}$ evolution with strain is very similar for both considered textures.

\subsection{Varied Twin Strength}

In this section, the initial strength of the extension twin mode was varied while the CRSS values for slip remained constant. The resulting changes in deformation behavior were examined for each of the preceding textures.

\subsubsection{Weak Basal Texture with Varied Twin Strength}

The deformation behavior of a weak basal texture with varied twin strength is shown in Figure 12 Similar to the basal textures in Section 3.1 .1 the stress-strain curves and slip activity along the RD and TD 
are nearly identical due to the symmetry of the basal peak. At tensile yield, the material with easiest twin activation has the lowest flow stress due to the twin activity; the material with increased CRSS for twinning has a higher flow stress because it must rely on the harder prismatic slip mode to accommodate deformation at and shortly after yield.

The starting texture, deformed textures, and pole figure difference plots as a function of twin strength are presented in Figure 13 for a uniaxial tension test along the sheet TD to a strain of 20\%. All three textures undergo basal peak spreading toward the TD and basal peak splitting toward the RD. Additionally, all three twin strengths exhibit strengthening of the sixfold symmetry of the prismatic pole figure, with the prism pole preferentially aligning toward the RD. The differences in texture evolution with the varied twin strength are subtle, but easier twin activation resulted in more basal poles aligned with the sheet TD as a result of the associated crystallographic reorientations.

Twinning is exhausted after less than $10 \%$ strain. Because twinning from TD tilt orientations reorients the crystal so that prismatic slip is favorable, a sharp increase in this slip mode is observed as twin activity diminishes for the intermediate and weak twin strengths. Prismatic slip plateaus at approximately 0.6 relative mode activity. A more gradual increase in prismatic slip activity and a lower plateau value in the absence of extension twinning is observed for the material with the highest CRSS for twinning. Decreased overall twin activity is also correlated with increased basal slip activity.

For this weak basal texture, all three twin strengths result in r-values after 10\% strain near 1.0 for all orientations within the sheet plane. Increased difficulty of twinning is correlated with lower r-values, suggesting improved formability. The values of $\bar{r}$ increase with strain for all twin strengths, but increase more rapidly with easier twin activation.

\subsubsection{Planar Anisotropy with Varied Twin Strength}

The deformation behavior for a material with a strongly anisotropic basal peak is shown in Figure 14 for the three considered CRSS values for extension twinning. In tension along the RD, the narrow spread of the c-axes orientations inhibits twin activity. As such, the deformation of all three twin strengths is nearly identical in this orientation. When straining in tension along the TD, the broader spread in c-axis orientation among the grains exaggerates the effects observed for the weak basal texture discussed in Section 3.2.1 With greater propensity for twinning, the flow stress at yield decreases, but increases rapidly as twinning is exhausted, resulting in an ultimately higher flow stress for strains greater than $8 \%$.

The texture evolution for uniaxial tension along the TD to a strain of $20 \%$ as a function of twin strength is shown in Figure 15. TD spreading and RD splitting of the basal peak is observed, qualitatively similar to 
the evolution described in Section 3.2.1 However, the basal peaks remain stronger during deformation, with a lesser amount of TD spreading occurring than in the preceding section. The trend of easy twin activation promoting the retention of TD spread remains.

The r-values for all orientations in the sheet plane increase with easier twin activation, but the variation of r-value decreases for easy twinning. The r-values of easily twinned material also evolve more rapidly with strain than material with more difficult twin activation. Both of these effects can be attributed to the rapid texture evolution caused by twinning, in comparison to the slower texture evolution in the presence of slip alone.

\subsubsection{Prismatic Plane Radial Isotropy with Varied Twin Strength}

Simulations were performed for the three considered twin strengths for a basal texture with a radially isotropic prism plane distribution. These results did not differ substantially from the results of the basal texture with sixfold symmetry in the prism plane distribution discussed in Section 3.2.1, therefore, they will not be presented here.

\section{Discussion}

A VPSC polycrystal plasticity simulation was used to evaluate r-value indicators of formability as a function of texture for an AZ61L magnesium alloy. The Voce hardening parameters used in the model were selected such that the hardening parameters match the in-plane plastic anisotropy, qualitative texture evolution, and experimental r-values of AZ61L sheet. Using the parameterized model, the effects of basal peak strength and symmetry, as well as prismatic plane distribution on deformation behavior was examined. Based on this analysis, basal peak strength has the strongest effect on forming behavior by a wide margin, with the other texture characteristics having only minor effects. Additionally, the effect of strength of the extension twinning deformation mode on forming behavior is evaluated by varying the CRSS for twinning while keeping the hardening parameters for all other deformation modes constant. For all examined textures, more tensile twinning resulted in increased simulated r-values, suggesting reduced formability.

For both symmetrical and asymmetrical basal peaks, easy twin activation at and shortly after tensile yield is replaced by prismatic slip as twinning is exhausted. Conversely, in the absence of twinning, greater basal slip is observed. Increased basal slip and decreased reliance on prismatic slip correlates with lower r-values, indicating improved formability. 


\subsection{Critical Resolved Shear Stress (CRSS) Ratios}

The selection of appropriate CRSS values for each deformation mode is a critical step in polycrystal plasticity simulations because the ratio of CRSS values for each mode determine the relative activity of deformation modes, which in turn determines texture evolution and macroscopic shape change. The r-value measures of formability measured in this study are a direct result of the macroscopic shape change, and will be dependent on the CRSS ratios. The observed ratios will depend on the true CRSS ratios, as well as other factors including grain size and shape or the presence of intermetallic particles [61-64]. Additional variation will result from the methods employed for determining the CRSS values.

In conventional magnesium alloys, the reported ratios depend much more sensitively on the measurement method than on the specific alloy. Some early single crystal studies reported basal:prismatic CRSS ratios approaching 1:100, even under constrained (plane strain compression) loading conditions designed to promote non-basal slip [65]. More recent polycrystal work has suggested ratios in the range of 1:1.1 to 1:8. Various reported ratios from the literature are presented in Table 3 The ratio used in this study, 1:2.6, falls within the reported range for polycrystal studies.

Basal slip:tension twin CRSS ratios reported in the literature are similarly varied, depending on both grain size and alloy content. Stanford and Barnett [61] report experimentally determined CRSS ratios for binary Mg-Zn alloys between 1:1.3-2.2. Raeisinia and Agnew [62] discuss ratios primarily in the range of 1:1.5-3 for binary Mg-Zn alloys with varied coarse grain sizes. Raeisinia et al. [63] review CRSS ratios determined for AZ31 via polycrystal plasticity modeling techniques, with values ranging from 1:0.7-33 reported, but most values are near 1:1.8. The ratios used in this work are 1:1.1, 1:1.8, and 1:3.2, within the commonly determined range.

Basal:pyramidal CRSS ratios are less commonly reported in the literature due to the difficulty in experimental measurements. Experimental studies based on single crystals report very high CRSS ratios, approaching 1:100 [66]. Similar VPSC polycrystal plasticity studies have found ratios near those determined in this work, near 1:3 [13, 67], though other polycrystal plasticity models have resulted in values greater than $1: 8$ [63].

\subsection{Texture and $r$-value behavior}

For all of the examined texture characteristics, the predicted r-values correlated strongly with the activity of prismatic slip. As discussed by Koike et al. [69], in the context of a tensile test of a sheet with a basal texture, width strains are primarily accommodated by prismatic slip while thickness strains are accommodated by pyramidal and to a lesser extent by basal slip. As such, high r-values-and the associated poor 
Table 3: Room temperature CRSS ratios reported in the literature for conventional alloys

\begin{tabular}{|c|c|c|c|c|c|}
\hline \multirow[t]{2}{*}{ Alloy } & \multicolumn{3}{|c|}{ CRSS Normalized to Basal } & \multirow[t]{2}{*}{ Single or Polycrystal } & \multirow[t]{2}{*}{ Ref. } \\
\hline & Prism. & Pyr. & Twin & & \\
\hline AZ31 & 1.1 & & & polycrystal & 68] \\
\hline AZ61 & $1.5-2$ & & & polycrystal & 69] \\
\hline AZ31B & $2-2.5$ & $2.4-3$ & & polycrystal & [26] \\
\hline AZ31B & 2.4 & 2.9 & 0.3 & polycrystal & [13] \\
\hline AZ61L & 2.6 & 3.7 & $1.1,1.8,3.2$ & polycrystal & present study \\
\hline $\mathrm{Mg}-0-3 \mathrm{wt} \% \mathrm{Zn}$ & $2.5-8$ & & $1.3-2.2$ & polycrystal & [61] \\
\hline $\mathrm{Mg}-0-2.3 \mathrm{wt} \% \mathrm{Zn}$ & & & $1.5-3$ & polycrystal & {$[62$} \\
\hline $\mathrm{AZ31B}$ & & 3 & 0.5 & polycrystal & {$[13,67]$} \\
\hline $\mathrm{Mg}-1.1 \mathrm{wt} \% \mathrm{Zn}$ & 30 & & & single crystal & [70, 71] \\
\hline $\mathrm{Mg}-0.5 \mathrm{wt} \% \mathrm{Zn}$ & 41 & & & single crystal & [70, 71 \\
\hline pure $\mathrm{Mg}$ & 50 & & & single crystal & [72] \\
\hline $\mathrm{Mg}-0.2 \mathrm{wt} \% \mathrm{Zn}$ & 53 & & & single crystal & [70,71] \\
\hline pure $\mathrm{Mg}$ & 90 & & & single crystal & [70, 71] \\
\hline pure $\mathrm{Mg}$ & $48-96$ & & & single crystal & 65] \\
\hline pure $\mathrm{Mg}$ & & 73 & & single crystal & 66] \\
\hline
\end{tabular}

formability - result from large amounts of prismatic slip relative to the activity of other deformation modes. The tradeoff between prismatic slip and basal slip is almost entirely determined by the basal pole tilt (angle between the grain c-axis and the sheet normal direction). This geometric tradeoff is the underlying reason for the strong sensitivity of r-value to the peak intensity in the basal pole figure and the dominance of this effect over those arising from the prismatic pole distribution or asymmetry of the basal peak shape.

The determined CRSS ratios directly impact the amount of basal pole tilt required for the Schmid factor for basal slip to equal that of prismatic slip. As discussed in the previous section, the CRSS ratio has reported to be grain size dependent [61-64], so the sensitivity of forming behavior to basal peak intensity may also be a function of grain size. The present results were parameterized based on fine-grained material, which other authors have correlated with enhancement of ductile behavior [10, 61, 68, 73, 74]. For more conventionally produced coarse grained material, the sensitivity to the strength of the basal peak may be even greater.

This insensitivity to the in-plane orientation of prismatic planes is in contrast to the results of the single crystal experiments performed by Kelley and Hosford [75]. That study examined the deformation behavior of $\mathrm{Mg}-4 \mathrm{wt} \% \mathrm{Li}$ in plane strain compression with the compression axis parallel to $\langle 10 \overline{1} 0\rangle$ versus $\langle 1 \overline{2} 10\rangle$ and constraint along the c-axis, both configurations to promote prismatic slip. They found that the differences in a-axis orientation resulted in substantially different strain hardening behavior as a result of differences in geometric hardening caused by the lattice rotation during deformation. In the present polycrystal work, 
these differences in geometric hardening behavior are not observed, likely because of the effect of averaging over 10,000 grains. Comparison with the later polycrystal results of Kelley and Hosford [12] is not possible because the authors only report the basal pole figures.

To summarize, the effect of texture on forming behavior is dominated by the strength of the basal peak, with the distribution of prismatic planes and basal peak symmetry having comparatively weak effects. Textures with high basal intensity deform with large amounts of prismatic slip, resulting in high r-values and poor forming behavior. The effects of prismatic plane distribution are largely averaged out by polycrystal effects. While asymmetry of the basal peak does result in differences in forming behavior, the strength of the peak remains the strongest effect.

\subsection{Twin mode strength and $r$-value behavior}

Changing the CRSS required for extension twinning produced substantial differences in predicted formability. For symmetrical and asymmetrical singly-peaked basal starting textures, easily-twinned material demonstrated larger r-values, suggesting reduced formability, because of the increased dependence on prismatic slip after reorientation of the grains. Conversely, inhibition of extension twinning resulted in lower r-values and therefore better predicted formability. This observation offers insight into alloying and microstructural design strategies which have the potential to enhance forming behavior. Reported strategies for inhibition extension twinning include alloying with rare earth elements and grain refinement, each of which is discussed in detail below.

In fine-grained materials $(\mathrm{d}<10 \mu \mathrm{m})$ with enhanced formability, some authors have attributed the enhancement to the activation of non-basal slip systems due to grain boundary constraint (e.g. [18, 26, 68]). Many of these studies have relied on transmission electron microscopy (TEM) studies of deformation mode activity performed at low strains. However, the fine grain size in these studies may also be inhibiting the activity of extension twinning [19--24, 64, 76, 77]. The simulations in the present work suggest that finegrained material with reduced twin activity would exhibit enhanced non-basal (prismatic) slip at low strains considered in most TEM investigations. However, the increase in prismatic slip is not the underlying reason for enhanced formability. In the current study, the lower r-values with inhibited twinning are the result of the continuing availability of basal slip as strain increases, instead of the rapid transition to dominant prismatic slip necessary when twinning is exhausted. This effect would be challenging to identify via TEM due to the difficulty of imaging highly deformed material.

An additional strategy to strengthen the extension twinning mode is via alloying additions. Non-RE alloy additions are generally reported to be relatively ineffective in strengthening extension twinning relative 
to other deformation modes [15, 61]. Conversely, rare earth additions, particularly highly-soluble additions such as $\mathrm{Y}$ and $\mathrm{Gd}$, have been reported to dramatically strengthen the extension twin relative to other deformation modes [14, 15, 78]. However, RE additions are also commonly reported to alter the activation of other twin modes, including compression and double twins [14, 17, 18]. Further analysis would be required to isolate the favorability of the activity of these twin types, which is beyond the scope of this work.

\subsection{The importance of prismatic slip activity}

The results of this work strongly suggest that poor formability, as predicted by high simulated r-values, is correlated with strong activity of prismatic slip after intermediate and high strains. However, the desirability of prismatic slip for forming behavior is disputed in the literature. This is largely because magnesium material that exhibits high elongation to failure in uniaxial tensile tests does not necessarily exhibit good formability [26].

Alloying and thermomechanical processing may be used to alter the balance between deformation modes, tailoring the mechanical properties. Many common solute strengthening additions for magnesium alloys, including $\mathrm{Al}$ and $\mathrm{Zn}$, are reported to soften prismatic slip while hardening against basal slip, effectively reducing the basal:prismatic CRSS ratio [61, 70, 71, 79]. The present results show that this solute effect may or may not be desirable, depending on the propensity for twinning of the material. Above the solubility limit, different alloying elements will form precipitates of different morphologies [2] (e.g. plates on prismatic versus basal planes or c-axis rods). These precipitate morphologies have different strengthening effects on each deformation mode based on their likelihood of interaction with particular types of dislocations or twins [80, 81]. Additionally, both solute and precipitate strengthening effects will be temperature sensitive; however, examining the temperature sensitivity is beyond the scope of this work.

The presence or absence of prismatic dislocations during and following deformation may have additional implications for recrystallization behavior. Dogan et al. [82] report that greater prismatic slip activity is observed in AZ31 material that resists shear band formation during equal channel angular pressing, in comparison to material where profuse twinning leads to dynamic recrystallization, which in turn leads to shear band formation. These authors suggest, therefore, that prismatic slip is a desirable alternative to profuse twinning under these conditions. Hadorn et al. [83] discuss the possibility that populations of dislocations on two orthogonal slip systems, such as basal and prismatic systems, may give subgrains additional degrees of rotational freedom during recrystallization, leading to weakening of the basal texture, which would in turn enhance formability. The effects of shear band formation and dynamic recrystallization would not be be captured by the VPSC model considered in this work. 


\subsection{Limiting factors for formability}

As shown in Figure 11, the limiting dome height in Erichsen tests decreases with increasing $\bar{r}$. This trend has been previously noted in magnesium and is also observed in other hcp metals, including zirconium alloys [84]. This is surprising, because a high $\bar{r}$ value in steels and aluminum alloys indicates that sheet material resists thinning during deformation, indicating good deep drawing behavior [42, 85]. This discrepancy suggests that, at least in certain regimes, the limiting dome height of hcp materials is not necessarily determined by a plastic instability (i.e. excessive sheet thinning), but by the initiation of fracture processes when thinning is required. This is unsurprising in sheet material with a basal texture due to the often discussed unavailability of deformation mechanisms along the c-axis [2, 13, 26]. However, Kang et al. demonstrate a correlation between the work hardening exponent and the limiting dome height for a range of both RE and non-RE containing alloys [36]. This indicates that there is a second failure regime where plastic instability is the dominant factor.

The influence of microstructure and alloying on the trade off between fracture and plastic instability remains to be clarified; however, it has been demonstrated that stress triaxiality plays an important role in conventional alloys [86-88]. Other authors report a brittle to ductile transition with grain refinement [10, 89, 90]. While coarse intermetallic particles have frequently been reported as failure initiation sites [86], Berman et al. show no decrease in tensile ductility with intermetallic particle phase fraction for a relatively homogeneous dispersion of $1 \mu \mathrm{m}$ particles [10]. This study highlights indicators of a susceptibility to premature failure in the fracture-dominated regime as predicted by r-values. Additional work is needed to first understand the transition to the plastic instability-dominated regime and subsequently determine how alloying and processing can be optimized to favor this second regime.

\section{Conclusions}

A viscoplastic self-consistent (VPSC) polycrystal plasticity model has been utilized to isolate the effects of extension twin CRSS values and texture characteristics common in magnesium alloys on their formability, as measured by $\mathrm{r}$-values. The VPSC model hardening parameters were selected using tensile data on thixomolded and thermomechanically processed AZ61L. These hardening parameters were used to simulate mechanical tests on simulated textures generated using DREAM.3D. In particular, the effects of basal peak intensity, distribution of prismatic plane directions, and symmetry of the basal peak are examined. The modeled results suggest the following: 
- A properly parameterized VPSC model is able to predict r-value evolution, providing insight into potential forming behavior as a function of texture and twin strength.

- Of the texture characteristics considered in the present study, the basal peak intensity is the best predictor of r-value behavior.

- Increasing basal peak strength requires the activation of additional prismatic slip during deformation. High prismatic slip activity results in large r-values and suggests poor formability.

- At constant basal peak strength, polycrystal tensile behavior is nearly insensitive to the effects of in-plane orientation of the prismatic planes and asymmetry of the peak in the basal pole figure.

- Lowering the CRSS for twinning results in larger r-values due to the rapid increase in prismatic slip activity as twinning reorients the crystal.

\section{Acknowledgments}

The authors would like to express their gratitude to Ray Decker and nanoMAG, LLC for providing the material used in this study. V.M. Miller additionally thanks the University of California Santa Barbara for the support of a Regents' Special Fellowship and T.M. Pollock acknowledges the support of Office of Naval Research Grant number N00014-12-1-0399. I.J. Beyerlein gratefully acknowledges support by a Laboratory Directed Research and Development program 20140348ER.

\section{References}

[1] D. Raabe, P. Klose, B. Engl, K.-P. Imlau, F. Friedel, and F. Roters. Concepts for integrating plastic anisotropy into metal forming simulations. Advanced Engineering Materials, 4(4):169, 2002.

[2] I. Polmear. Light Alloys: From Traditional Alloys to Nanocrystals. Butterworth-Heinemann, Burlington, MA, 4 edition, 2006.

[3] J. Bhattacharyya, S. Agnew, and G. Muralidharan. Texture enhancement during grain growth of magnesium alloy AZ31b. Acta Materialia, 86:80-94, March 2015.

[4] I. Basu, T. Al-Samman, and G. Gottstein. Shear band-related recrystallization and grain growth in two rolled magnesium-rare earth alloys. Materials Science and Engineering: A, 579:50-56, September 2013. 
[5] M. Steiner, J. Bhattacharyya, and S. Agnew. The origin and enhancement of $\{0001\}<11-20>$ texture during heat treatment of rolled AZ31B magnesium alloys. Acta Materialia, 95:443-455, August 2015.

[6] R. Gehrmann, M. M. Frommert, and G. Gottstein. Texture effects on plastic deformation of magnesium. Materials Science and Engineering: A, 395(1-2):338-349, March 2005.

[7] S. Agnew, J. Horton, T. Lillo, and D. Brown. Enhanced ductility in strongly textured magnesium produced by equal channel angular processing. Scripta Materialia, 50(3):377-381, February 2004.

[8] N. Stanford and M. Barnett. The origin of "rare earth" texture development in extruded Mg-based alloys and its effect on tensile ductility. Materials Science and Engineering: A, 496(1-2):399-408, November 2008.

[9] N. Stanford, D. Atwell, A. Beer, C. Davies, and M. Barnett. Effect of microalloying with rare-earth elements on the texture of extruded magnesium-based alloys. Scripta Materialia, 59(7):772-775, October 2008.

[10] T. D. Berman, T. M. Pollock, and J. W. Jones. Texture, Second-Phase Particles, and the Anisotropy of Deformation Behavior in TTMP AZ61. Metallurgical and Materials Transactions A, 46(7):2986-2998, July 2015.

[11] Y. Wang and H. Choo. Influence of texture on Hall-Petch relationships in an Mg alloy. Acta Materialia, 81:83-97, December 2014.

[12] E. Kelley and W. Hosford. The Deformation Characteristics of Textured Magnesium. Transactions of the Metallurgical Society of AIME, 242(4):654-661, 1968.

[13] S. R. Agnew. Plastic Anisotropy of Magnesium Alloy AZ31b Sheet. In Magnesium Technology 2002, pages 351-356, 2002.

[14] N. Stanford, R. Marceau, and M. Barnett. The effect of high yttrium solute concentration on the twinning behaviour of magnesium alloys. Acta Materialia, 82:447-456, January 2015.

[15] J. F. Nie, Y. M. Zhu, J. Z. Liu, and X. Y. Fang. Periodic Segregation of Solute Atoms in Fully Coherent Twin Boundaries. Science, 340(6135):957-960, May 2013.

[16] I. Basu and T. Al-Samman. Twin recrystallization mechanisms in magnesium-rare earth alloys. Acta Materialia, 96:111-132, September 2015. 
[17] K. Hantzsche, J. Bohlen, J. Wendt, K. Kainer, S. Yi, and D. Letzig. Effect of rare earth additions on microstructure and texture development of magnesium alloy sheets. Scripta Materialia, 63(7):725730, October 2010.

[18] S. Sandlöbes, S. Zaefferer, I. Schestakow, S. Yi, and R. Gonzalez-Martinez. On the role of non-basal deformation mechanisms for the ductility of Mg and Mg-Y alloys. Acta Materialia, 59(2):429-439, January 2011.

[19] C. Cepeda-Jiménez, J. Molina-Aldareguia, and M. Pérez-Prado. Origin of the twinning to slip transition with grain size refinement, with decreasing strain rate and with increasing temperature in magnesium. Acta Materialia, 88:232-244, April 2015.

[20] M. Barnett, Z. Keshavarz, A. Beer, and D. Atwell. Influence of grain size on the compressive deformation of wrought Mg-3Al-1Zn. Acta Materialia, 52(17):5093-5103, October 2004.

[21] P. Dobroň, F. Chmelík, S. Yi, K. Parfenenko, D. Letzig, and J. Bohlen. Grain size effects on deformation twinning in an extruded magnesium alloy tested in compression. Scripta Materialia, 65(5):424-427, September 2011.

[22] J. Li, W. Xu, X. Wu, H. Ding, and K. Xia. Effects of grain size on compressive behaviour in ultrafine grained pure $\mathrm{Mg}$ processed by equal channel angular pressing at room temperature. Materials Science and Engineering: A, 528(18):5993-5998, July 2011.

[23] H. Choi, Y. Kim, J. Shin, and D. Bae. Deformation behavior of magnesium in the grain size spectrum from nano- to micrometer. Materials Science and Engineering: A, 527(6):1565-1570, March 2010.

[24] Y. Chino, K. Kimura, and M. Mabuchi. Twinning behavior and deformation mechanisms of extruded AZ31 Mg alloy. Materials Science and Engineering: A, 486(1-2):481-488, July 2008.

[25] W. Lankford, S. Snyder, and J. Bauscher. New criteria for predicting the press performance of deep drawing sheets. Transactions of the American Society for Metals, 42:1197-1232, 1950.

[26] S. R. Agnew and Ö. Duygulu. Plastic anisotropy and the role of non-basal slip in magnesium alloy AZ31b. International Journal of Plasticity, 21(6):1161-1193, June 2005.

[27] X. Huang, K. Suzuki, and N. Saito. Textures and stretch formability of Mg-6Al-1Zn magnesium alloy sheets rolled at high temperatures up to 793K. Scripta Materialia, 60(8):651-654, April 2009. 
[28] D. Wu, R. Chen, and E. Han. Excellent room-temperature ductility and formability of rolled Mg-Gd-Zn alloy sheets. Journal of Alloys and Compounds, 509(6):2856-2863, February 2011.

[29] Y. Chino, K. Sassa, and M. Mabuchi. Enhanced stretch formability of Mn-free AZ31 Mg alloy rolled by cross-roll rolling. Journal of Materials Science, 44(7):1821-1827, April 2009.

[30] X. Huang, K. Suzuki, Y. Chino, and M. Mabuchi. Improvement of stretch formability of Mg-3Al-1Zn alloy sheet by high temperature rolling at finishing pass. Journal of Alloys and Compounds, 509(28):7579-7584, July 2011.

[31] Y. Chino and M. Mabuchi. Enhanced stretch formability of $\mathrm{Mg}-\mathrm{Al}-\mathrm{Zn}$ alloy sheets rolled at high temperature (723k). Scripta Materialia, 60(6):447-450, March 2009.

[32] E. Yukutake, J. Kaneko, and M. Sugamata. Anisotropy and non-uniformity in plastic behavior of AZ31 magnesium alloy plates. Materials Transactions, 44(4):452-457, 2003.

[33] Y. Chino, H. Iwasaki, and M. Mabuchi. Stretch formability of AZ31 Mg alloy sheets at different testing temperatures. Materials Science and Engineering: A, 466(1-2):90-95, September 2007.

[34] X. Huang, K. Suzuki, A. Watazu, I. Shigematsu, and N. Saito. Improvement of formability of $\mathrm{Mg}-\mathrm{Al}-\mathrm{Zn}$ alloy sheet at low temperatures using differential speed rolling. Journal of Alloys and Compounds, 470(1-2):263-268, February 2009.

[35] H. Zhang, G. Huang, D. Kong, G. Sang, and B. Song. Influence of initial texture on formability of AZ31b magnesium alloy sheets at different temperatures. Journal of Materials Processing Technology, 211(10):1575-1580, October 2011.

[36] D. H. Kang, D. W. Kim, S. Kim, G. T. Bae, K. H. Kim, and N. J. Kim. Relationship between stretch formability and work-hardening capacity of twin-roll cast $\mathrm{Mg}$ alloys at room temperature. Scripta Materialia, 61(7):768-771, 2009.

[37] K. K. Mathur, P. Dawson, and U. Kocks. On modeling anisotropy in deformation processes involving textured polycrystals with distorted grain shape. Mechanics of Materials, 10:183-202, 1990.

[38] L. Delannay, M. Melchior, J. Signorelli, J.-F. Remacle, and T. Kuwabara. Influence of grain shape on the planar anisotropy of rolled steel sheets - evaluation of three models. Computational Materials Science, 45(3):739-743, May 2009. 
[39] D. R. Kumar. Formability analysis of extra-deep drawing steel. Journal of Materials Processing Technology, 130:31-41, 2002.

[40] F. Barlat and J. Liu. Precipitate-induced anisotropy in binary Al-Cu alloys. Materials Science and Engineering: A, A257:47-61, 1998.

[41] G. Winther, D. Juul Jensen, and N. Hansen. Modelling flow stress anisotropy caused by deformation induced dislocation boundaries. Acta Materialia, 45(6):2455-2465, 1997.

[42] S.-H. Choi, J. C. Brem, F. Barlat, and K. H. Oh. Macroscopic anisotropy in AA5019a sheets. Acta materialia, 48(8):1853-1863, 2000.

[43] G. Charca Ramos, M. Stout, R. Bolmaro, J. Signorelli, M. Serenelli, M. Bertinetti, and P. Turner. Study of a drawing-quality sheet steel. II: Forming-limit curves by experiments and micromechanical simulations. International Journal of Solids and Structures, 47(17):2294-2299, August 2010.

[44] H. Wang, P. Wu, K. Boyle, and K. Neale. On crystal plasticity formability analysis for magnesium alloy sheets. International Journal of Solids and Structures, 48(6):1000-1010, March 2011.

[45] J. Lévesque, K. Inal, K. Neale, and R. Mishra. Numerical modeling of formability of extruded magnesium alloy tubes. International Journal of Plasticity, 26(1):65-83, January 2010.

[46] C. Neil and S. R. Agnew. Crystal placicity-based formin limit prediction for non-cubic metals: Application to Mg alloy AZ31b. International Journal of Plasticity, 25:379-398, 2009.

[47] R. A. Lebensohn and C. Tomé. A self-consistent anisotropic approach for the simulation of plastic deformation and texture development of polycrytsals: application to zirconium alloys. Acta Metallurgica et Materialia, 41(9):2611-2624, 1993.

[48] C. N. Tomé. Self-consistent polycrystal models: a directional compliance criterion to describe grain interactions. Modelling and Simulation in Materials Science and Engineering, 7(5):723, 1999.

[49] T. D. Berman, W. Donlon, V. M. Miller, R. Decker, J. Huang, T. M. Pollock, and J. W. Jones. MICROSTRUCTURE MODIFICATION AND DEFORMATION BEHAVIOR OF FINE GRAINED AZ611 SHEET PRODUCED BY THIXOMOLDING® AND THERMOMECHANICAL PROCESSING. Magnesium Technology 2012 w/CD, page 339, 2012. 
[50] V. M. Miller, T. D. Berman, I. J. Beyerlein, and T. Pollock. Prediction of Formability in Magnesium Alloys: The Role of Texture. In Magnesium Technology 2016, volume Accepted, Nashville, TN, 2016. TMS (The Minerals, Metals \& Materials Society).

[51] C. N. Tome, G. Canova, U. Kocks, N. Christodoulou, and J. Jonas. The relation between macroscopic and microscopic strain hardening in F.C.C. polycrystals. Acta Metallurgica, 32(10):1637-1653, 1984.

[52] C. Tomé, R. A. Lebensohn, and U. Kocks. A model for texture development dominated by deformation twinning: applicatoin to zirconium Alloys. Acta Metallurgica et Materialia, 39(11):2667-2680, 1991.

[53] S. G. Vogel, D. J. Alexander, I. J. Beyerlein, M. A. Bourke, D. W. Brown, B. Clausen, C. Tomé, B. Von Dreele, C. Xu, and T. G. Langdon. Investigation of texture in ECAP materials using neutron diffraction. Materials Science Forum, 426-432:2661-2665, 2003.

[54] S. C. Vogel, I. J. Beyerlein, M. A. Bourke, C. Tomé, P. Rangaswamy, C. Xu, and T. G. Langdon. Texture in equal-channel angular pressed aluminum and nickel. Materials Science Forum, 408-412:673-678, 2002.

[55] J. S. Carpenter, R. J. McCabe, S. J. Zheng, T. A. Wynn, N. A. Mara, and I. J. Beyerlein. Processing Parameter Influence on Texture and Microstructural Evolution in $\mathrm{Cu}-\mathrm{Nb}$ Multilayer Composites Fabricated via Accumulative Roll Bonding. Metallurgical and Materials Transactions A, 45(4):2192-2208, April 2014.

[56] S. Li, I. J. Beyerlein, C. T. Necker, D. J. Alexander, and M. Bourke. Heterogeneity of deformation texture in equal channel angular extrusion of copper. Acta Materialia, 52(16):4859-4875, September 2004.

[57] S. Li, I. J. Beyerlein, D. J. Alexander, and S. C. Vogel. Texture evolution during multi-pass equal channel angular extrusion of copper: Neutron diffraction characterization and polycrystal modeling. Acta Materialia, 53(7):2111-2125, April 2005.

[58] M. A. Groeber and M. A. Jackson. DREAM. 3d: a digital representation environment for the analysis of microstructure in 3d. Integrating Materials and Manufacturing Innovation, 3(1):1-17, 2014.

[59] F. Bachmann, R. Hielscher, and H. Schaeben. Texture Analysis with MTEX - Free and Open Source Software Toolbox. Solid State Phenomena, 160:63-68, February 2010. 
[60] X. Li, F. Jiao, T. Al-Samman, and S. Ghosh Chowdhury. Influence of second-phase precipitates on the texture evolution of Mg-Al-Zn alloys during hot deformation. Scripta Materialia, 66(3-4):159-162, February 2012.

[61] N. Stanford and M. Barnett. Solute strengthening of prismatic slip, basal slip and twinning in Mg and Mg-Zn binary alloys. International Journal of Plasticity, 47:165-181, August 2013.

[62] B. Raeisinia and S. R. Agnew. Using polycrystal plasticity modeling to determine the effects of grain size and solid solution additions on individual deformation mechanisms in cast $\mathrm{Mg}$ alloys. Scripta Materialia, 63(7):731-736, October 2010.

[63] B. Raeisinia, S. R. Agnew, and A. Akhtar. Incorporation of Solid Solution Alloying Effects into Polycrystal Modeling of Mg Alloys. Metallurgical and Materials Transactions A, 42(5):1418-1430, May 2011.

[64] A. Jain, O. Duygulu, D. Brown, C. Tomé, and S. Agnew. Grain size effects on the tensile properties and deformation mechanisms of a magnesium alloy, AZ31b, sheet. Materials Science and Engineering: A, 486(1-2):545-555, July 2008.

[65] W. Hutchinson and M. Barnett. Effective values of critical resolved shear stress for slip in polycrystalline magnesium and other hcp metals. Scripta Materialia, 63(7):737-740, October 2010.

[66] T. Obara, H. Yoshinga, and S. Morozumi. $\{11-22\}<11-23>$ Slip system in magnesium. Acta Metallurgica, 21(7):845-853, 1973.

[67] S. R. Agnew, M. H. Yoo, and C. N. Tome. Application of texture simulation to understanding mechanical behavior of Mg and solid solution alloys containing Li or Y. Acta Materialia, 49(20):4277-4289, 2001.

[68] J. Koike, T. Kobayashi, T. Mukai, H. Watanabe, M. Suzuki, K. Maruyama, and K. Higashi. The activity of non-basal slip systems and dynamic recovery at room temperature in fine-grained AZ31b magnesium alloys. Acta materialia, 51(7):2055-2065, 2003.

[69] J. Koike and R. Ohyama. Geometrical criterion for the activation of prismatic slip in AZ61 Mg alloy sheets deformed at room temperature. Acta Materialia, 53(7):1963-1972, April 2005. 
[70] A. Akhtar and E. Teghtsoonian. Solid solution strengthening of magnesium single crystals - I. Alloying behaviour in basal slip. Acta Metallurgica, 17:1339-1349, 1969.

[71] A. Akhtar and E. Teghtsoonian. Solid Solution Strengthening of Magnesium Single Crystals-II The Effect of Solute on the Ease of Prismatic Slip. Acta Metallurgica, 17:1351-1356, 1969.

[72] A. Couret and D. Caillard. An in situ study of prismatic glide in magnesium-I. The rate controlling mechanism. Acta Metallurgica, 33(8):1447-1454, 1985.

[73] A. Hänzi, F. Dalla Torre, A. Sologubenko, P. Gunde, R. Schmid-Fetzer, M. Kuehlein, J. Löffler, and P. Uggowitzer. Design strategy for microalloyed ultra-ductile magnesium alloys. Philosophical Magazine Letters, 89:377-390, June 2009.

[74] A. Yamashita, Z. Horita, and T. G. Langdon. Improving the mechanical properties of magnesium and a magnesium alloy through severe plastic deformation. Materials Science and Engineering: A, 300(1):142-147, 2001.

[75] E. Kelley and W. Hosford. Plane strain compression of Mg and Mg alloy single crystals. Trans. Metall. Soc. A.I.M.E., 242(1):5-13, 1968.

[76] H. Asgari, J. Szpunar, A. Odeshi, L. Zeng, and E. Olsson. Effect of grain size on high strain rate deformation of rolled Mg-4Y-3RE alloy in compression. Materials Science and Engineering: A, 633:92-102, May 2015.

[77] M. A. Meyers, O. Vöhringer, and V. A. Lubarda. The onset of twinning in metals: a constitutive description. Acta materialia, 49(19):4025-4039, 2001.

[78] M. Lentz, M. Klaus, M. Wagner, C. Fahrenson, I. J. Beyerlein, M. Zecevic, W. Reimers, and M. Knezevic. Effect of age hardening on the deformation behavior of an $\mathrm{Mg}-\mathrm{Y}-\mathrm{Nd}$ alloy: In-situ X-ray diffraction and crystal plasticity modeling. Materials Science and Engineering: A, 628:396-409, March 2015.

[79] H. Somekawa, M. Yamaguchi, Y. Osawa, A. Singh, M. Itakura, T. Tsuru, and T. Mukai. Material design for magnesium alloys with high deformability. Philosophical Magazine, 95(8):869-885, March 2015. 
[80] J. Nie. Effects of precipitate shape and orientation on dispersion strengthening in magnesium alloys. Scripta Materialia, 48:1009-1015, 2003.

[81] J. Robson, N. Stanford, and M. Barnett. Effect of precipitate shape on slip and twinning in magnesium alloys. Acta Materialia, 59(5):1945-1956, March 2011.

[82] E. Dogan, M. Vaughan, S. Wang, I. Karaman, and G. Proust. Role of starting texture and deformation modes on low-temperature shear formability and shear localization of $\mathrm{Mg}-3 \mathrm{Al}-1 \mathrm{Zn}$ alloy. Acta Materialia, 89:408-422, May 2015.

[83] J. P. Hadorn, K. Hantzsche, S. Yi, J. Bohlen, D. Letzig, J. A. Wollmershauser, and S. R. Agnew. Role of Solute in the Texture Modification During Hot Deformation of Mg-Rare Earth Alloys. Metallurgical and Materials Transactions A, 43(4):1347-1362, October 2011.

[84] J. Bohlen, M. R. Nürnberg, J. W. Senn, D. Letzig, and S. R. Agnew. The texture and anisotropy of magnesium-zinc-rare earth alloy sheets. Acta Materialia, 55(6):2101-2112, April 2007.

[85] W. Hosford. The Mechanics of Crystals and Textured Polycrystals. Number 32 in Oxford Engineering Science Series. Oxford University Press, New York, 1993.

[86] B. Kondori and A. A. Benzerga. Effect of Stress Triaxiality on the Flow and Fracture of Mg Alloy AZ31. Metallurgical and Materials Transactions A, 45(8):3292-3307, July 2014.

[87] B. Kondori and A. A. Benzerga. Fracture Strains, Damage Mechanisms and Anisotropy in a Magnesium Alloy Across a Range of Stress Triaxialities. Experimental Mechanics, 54(3):493-499, March 2014.

[88] B. Kondori and A. Benzerga. On the notch ductility of a magnesium-rare earth alloy. Materials Science and Engineering: A, 647:74-83, October 2015.

[89] T. Mukai, T. Mohri, M. Mabuchi, M. Nakamura, K. Ishikawa, and K. Higashi. Experimental study of a structural magnesium alloy with high absorption energy under dynamic loading. Scripta materialia, 39(9):1249-1253, 1998.

[90] Q. Miao, L. Hu, G. Wang, and E. Wang. Fabrication of excellent mechanical properties AZ31 magnesium alloy sheets by conventional rolling and subsequent annealing. Materials Science and Engineering: A, 528(22-23):6694-6701, August 2011. 
RD Uniaxial Tension
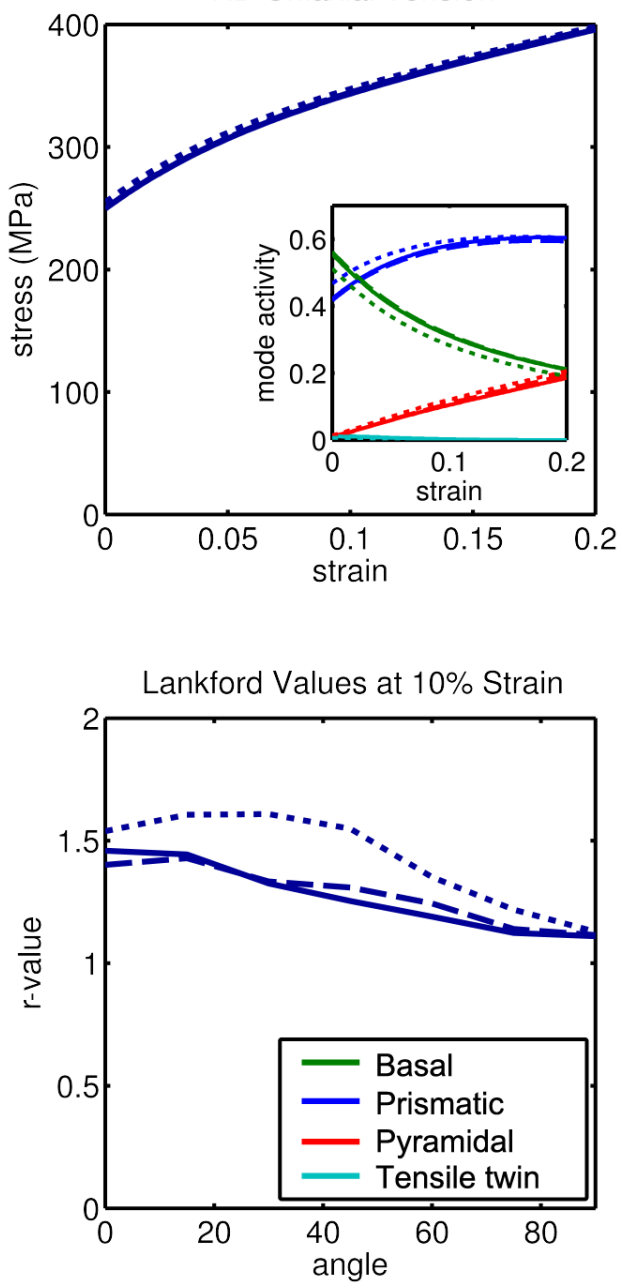

TD Uniaxial Tension

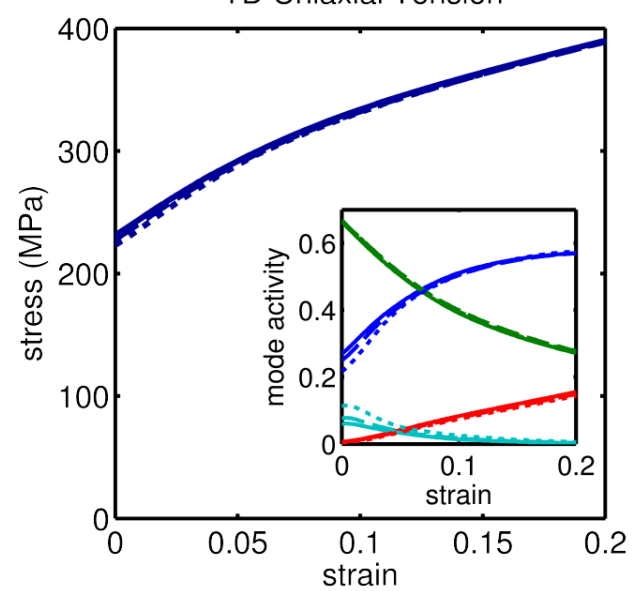

$\bar{r}$

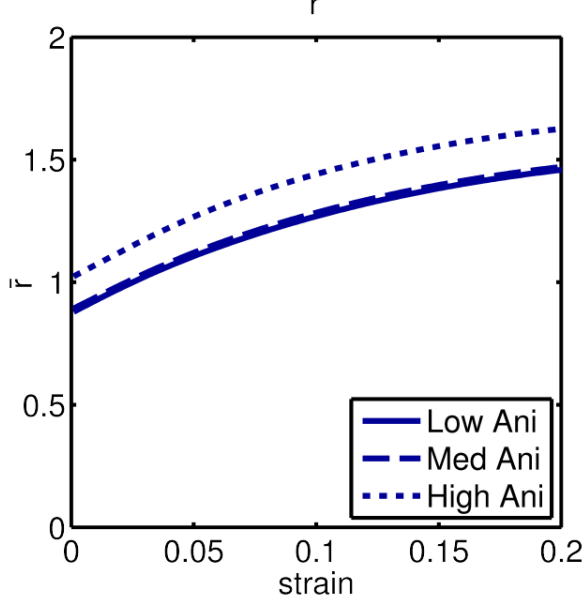

Figure 8: Changes in deformation behavior with varied in-plane anisotropy of c-axis orientation for fine-grained sheet. 


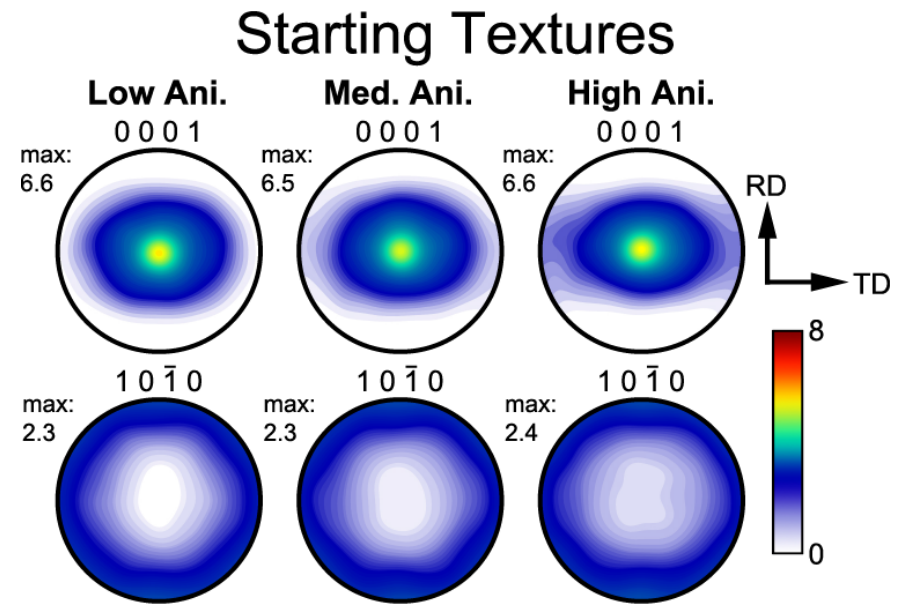

\section{Final Textures}

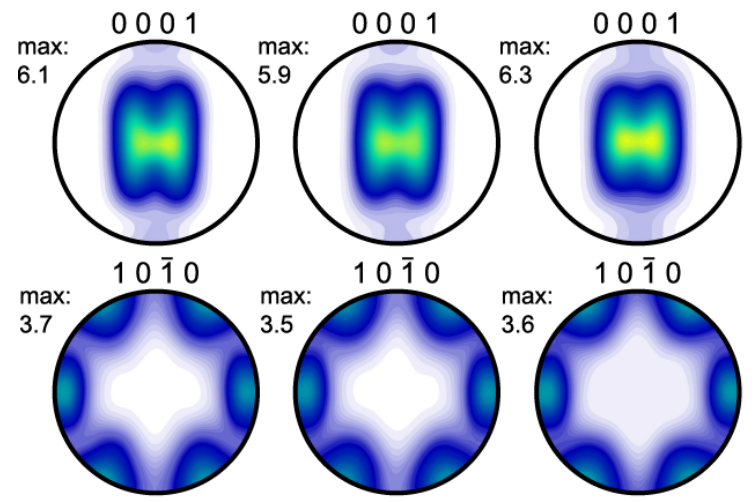

\section{PF Difference Maps}

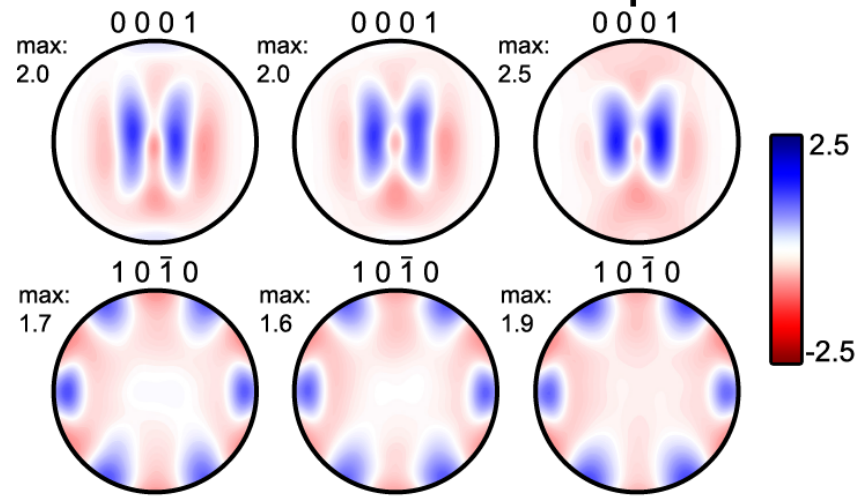

Figure 9: Texture evolution as a function of varied in-plane anisotropy of the c-axis distribution during uniaxial tension along the TD to $20 \%$ strain. The initial textures, deformed textures, and pole figure difference plots are shown. 
Starting Textures

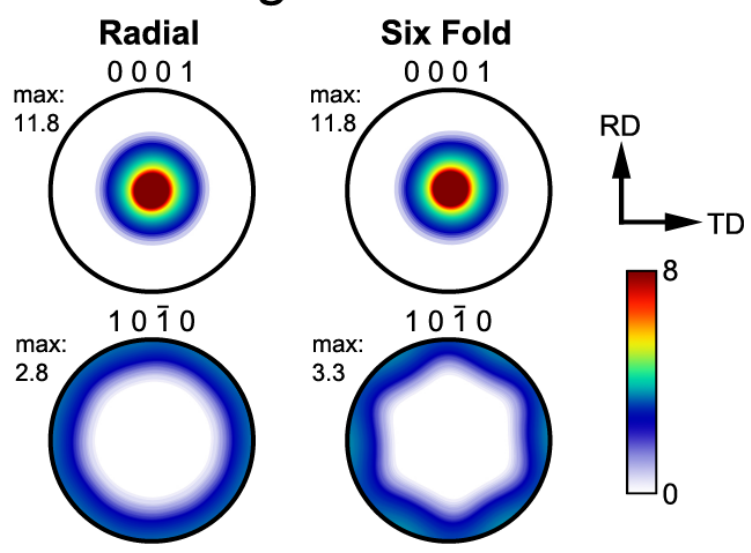

\section{Final Textures}
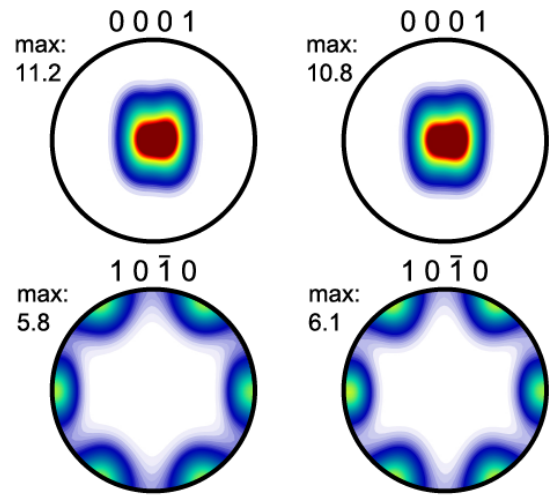

\section{PF Difference Maps}

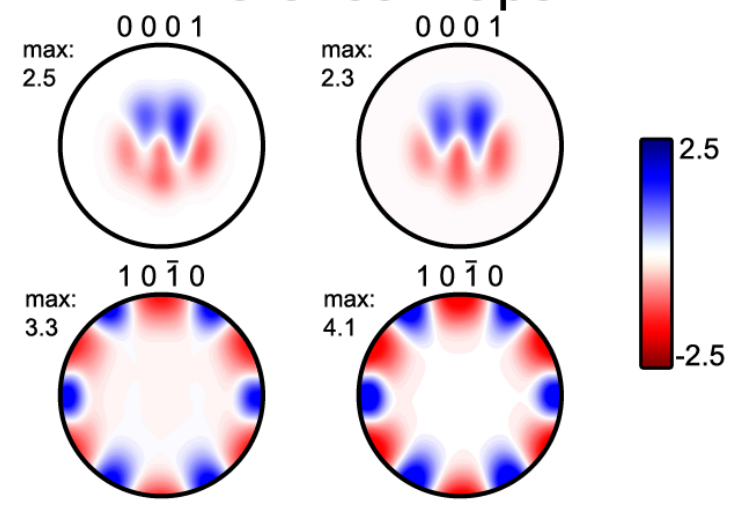

Figure 10: Texture evolution during uniaxial tension along the sheet TD to $20 \%$ strain for symmetrical basal textures as a function of in-plane a-axis alignment. The initial textures, deformed textures, and pole figure difference plots are shown. 
RD Uniaxial Tension
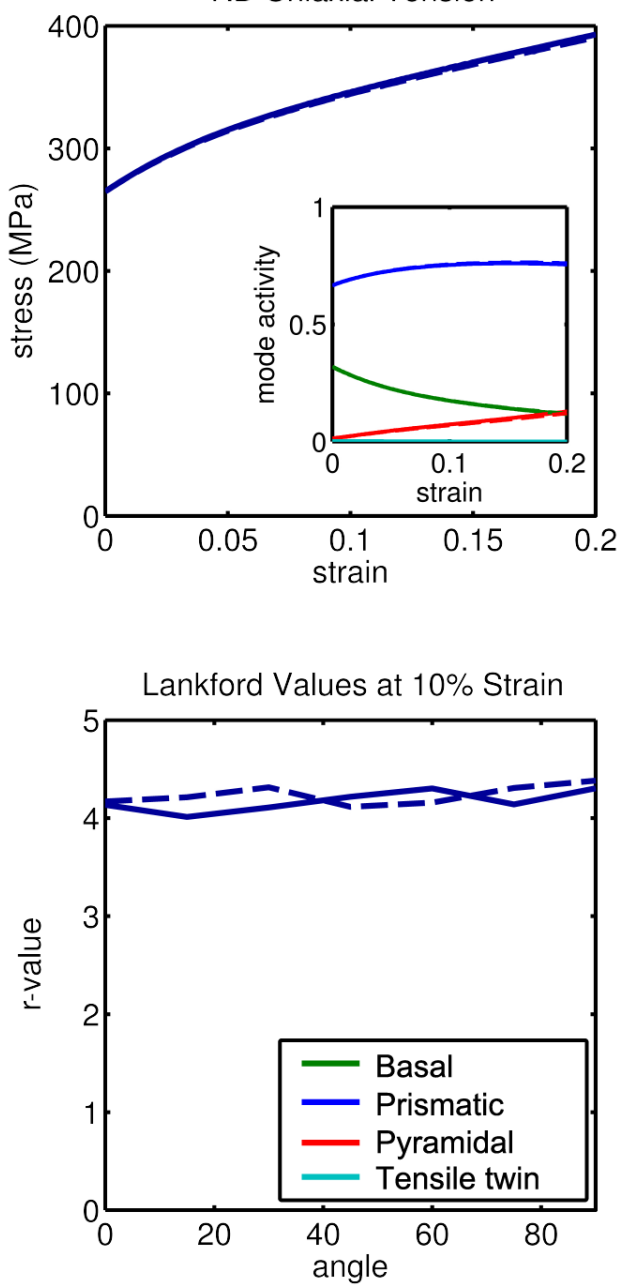

TD Uniaxial Tension
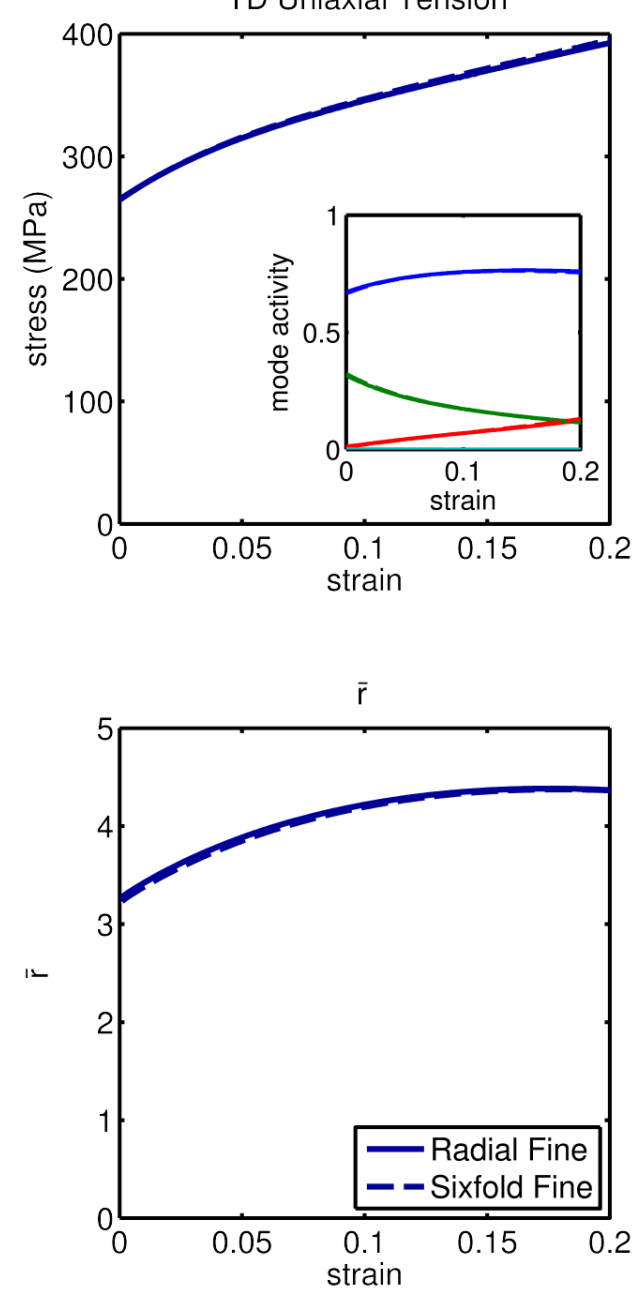

Figure 11: Altered deformation behavior with varied in-plane a-axis alignment for fine-grained sheet. 

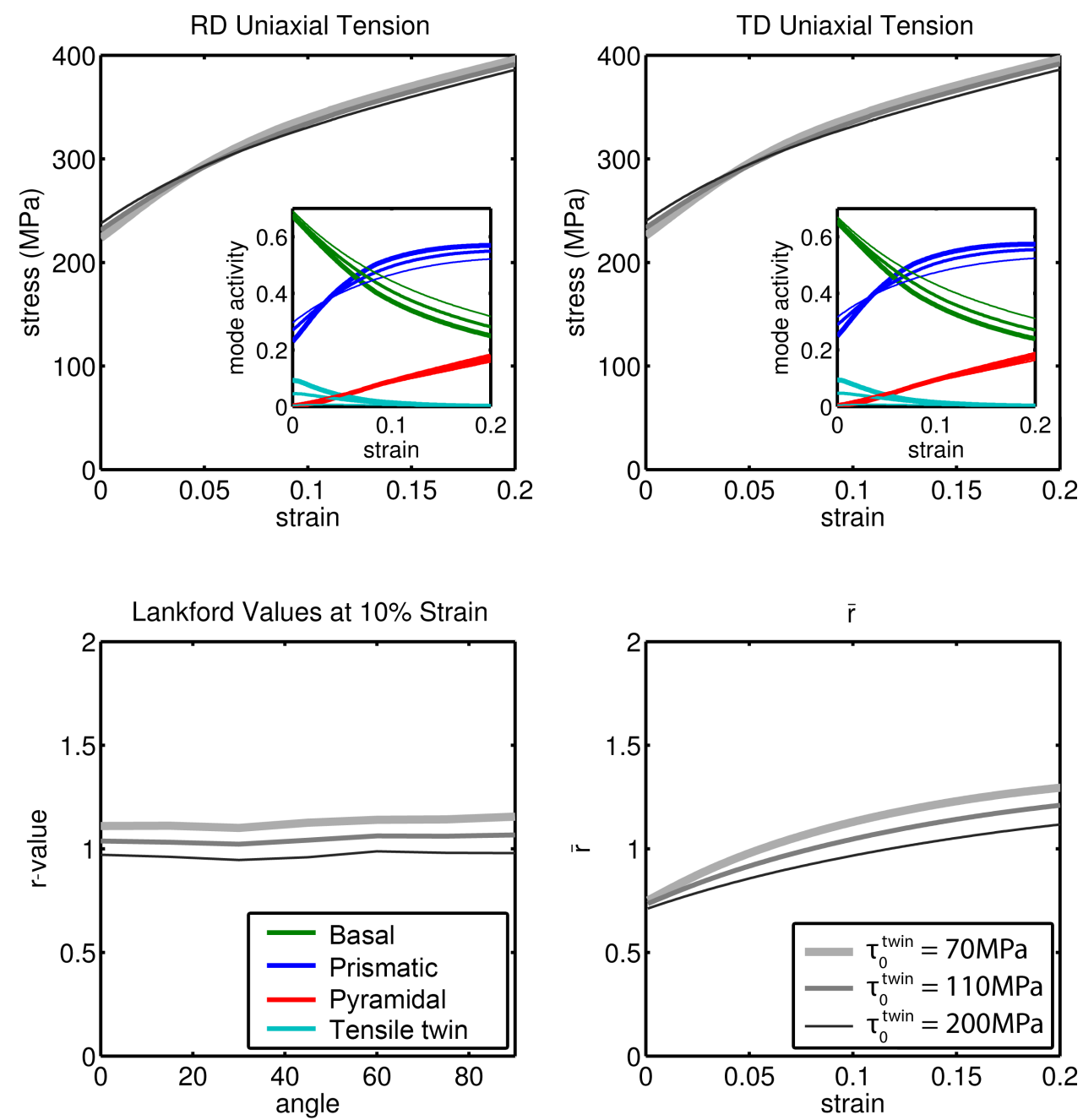

Figure 12: Deformation behavior for weak basal textures as a function of twin strength. 


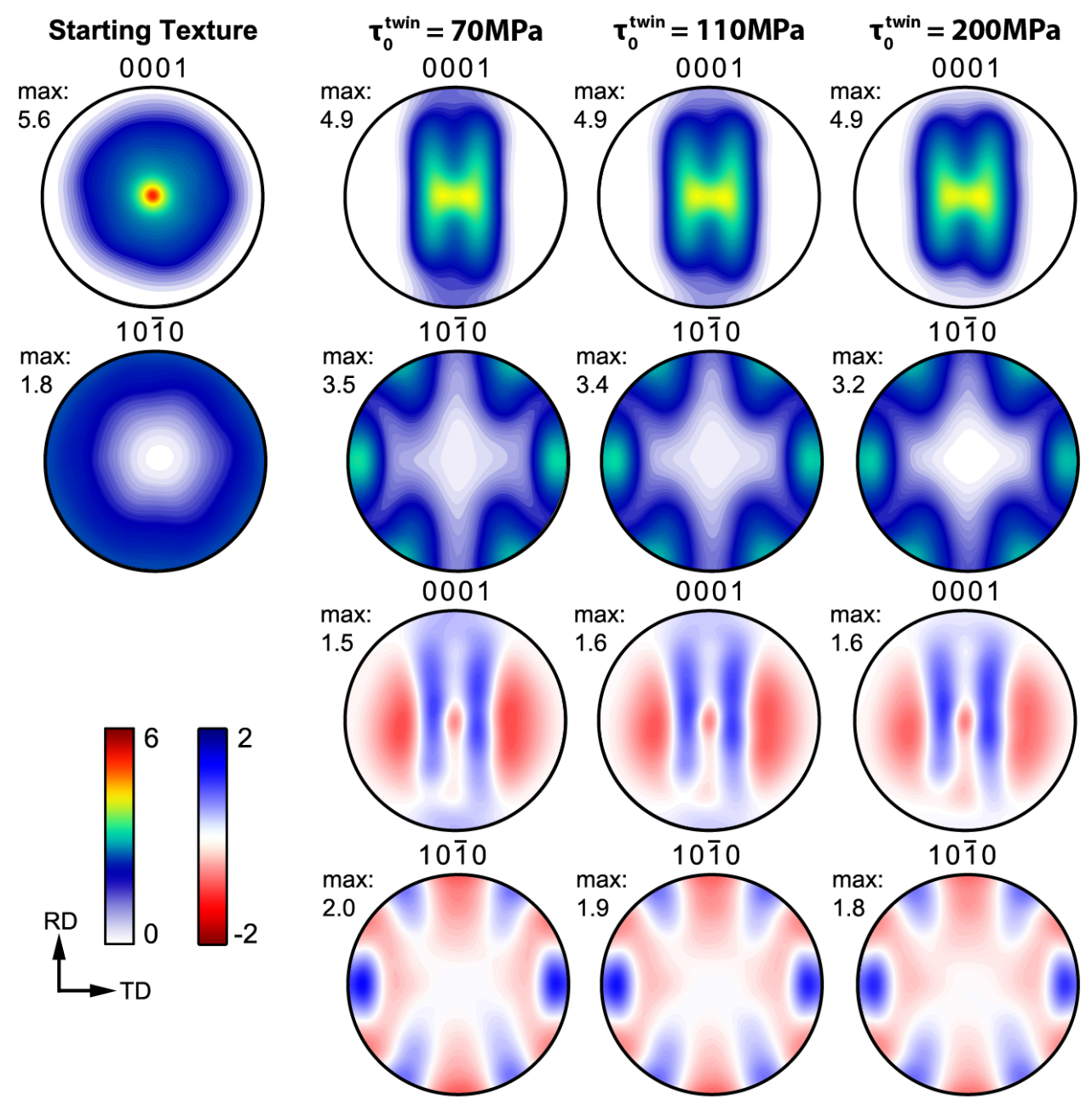

Figure 13: Evolution of a weak basal texture during uniaxial tension along the TD to a strain of $20 \%$ as a function of twin strength. The initial texture, final textures, and pole figure difference plots are shown. 

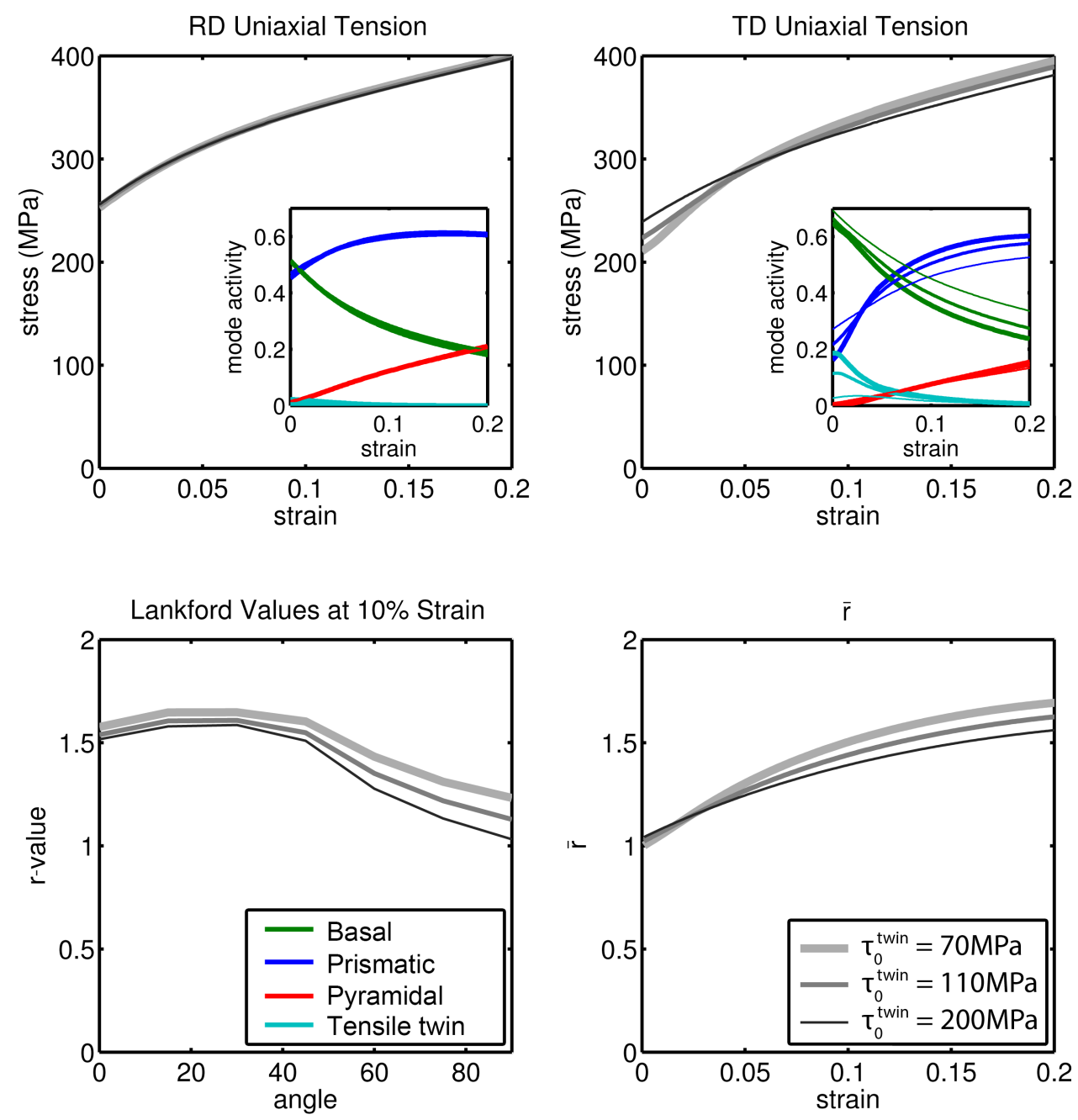

Figure 14: Deformation behavior as a function of twin strength for a strongly anisotropic basal peak. 


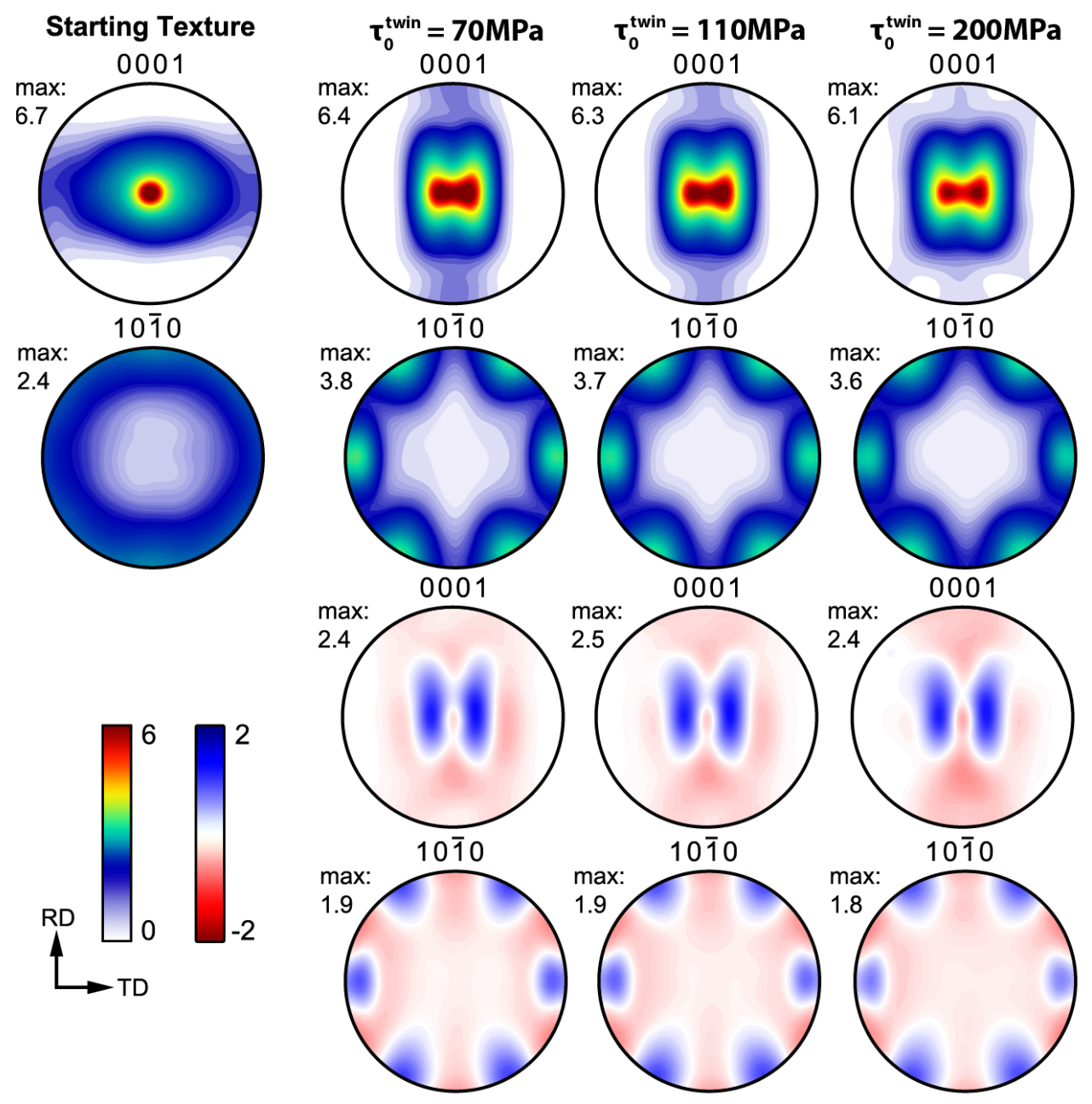

Figure 15: Texture evolution during uniaxial tension along the TD to strains of $20 \%$ as a function of twin strength for a strongly asymmetric basal peak. The initial texture, deformed textures, and pole figure difference maps are shown. 